\title{
GENERALIZED INTERPOLATION SPACES
}

\author{
BY \\ VERNON WILLIAMS
}

\begin{abstract}
In this paper we introduce the notion of "generalized" interpolation space, and state and prove a "generalized" interpolation theorem. This apparently provides a foundation for an axiomatic treatment of interpolation space theory, for subsequently we show that the "mean" interpolation spaces of Lions-Peetre, the "complex" interpolation spaces of A. P. Calderón, and the "complex" interpolation spaces of M. Schechter are all generalized interpolation spaces. Furthermore, we prove that each of the interpolation theorems established respectively for the abovementioned interpolation spaces is indeed a special case of our generalized interpolation theorem.

In §III of this paper we use the generalized interpolation space concept to state and prove a "generalized" duality theorem. The very elegant duality theorems proved by Calderón, Lions-Peetre and Schechter, respectively, are shown to be special cases of our generalized duality theorem. Of special interest here is the isolation by the general theorem of the need in each of the separate theorems for certain "base" spaces to be duals of others.

At the close of §II of this paper we employ our generalized interpolation theorem "structure" to construct new interpolation spaces which are neither complex nor mean spaces.
\end{abstract}

I. Introduction. Since the advent in 1926 of the classical interpolation theorem on $L^{p}$ spaces by Marcel Riesz (see [10]), the subject of interpolation between Banach spaces has developed quite rapidly.

A. P. Calderón has used Banach space valued functions on the strip in the complex plane together with the delta functional to construct his complex interpolation spaces, $\left(X_{0}, X_{1}\right)_{s}$. See [1].

Quite recently, M. Schechter has used Banach space valued functions defined in the complex strip together with a two-dimensional distribution $T$ with compact support to construct more general complex interpolation spaces, $\left(X_{0}, X_{1}\right)_{T}$, $\left(X_{0}, X_{1}\right)^{T}$, for which the above mentioned Calderón spaces are a special case. See [11].

Lions and Peetre have used Banach space valued "weighted" functions with real domain to construct their "Espaces de Moyennes", $S\left(p_{0}, r_{0}, X_{0} ; p_{1}, r_{1}, X_{1}\right)$. See [6].

Received by the editors September 26, 1969.

AMS 1969 subject classifications. Primary 4638; Secondary 4725, 4730.

Key words and phrases. Interpolation pair of Banach spaces, analytic vector-valued functions, Lebesgue integrable vector-valued functions, distributions with compact support, Calderón complex interpolation spaces, Lions-Peetre mean interpolation spaces, Schechter complex interpolation spaces, generalized interpolation spaces.

Copyright $(\subset)$ 1971, American Mathematical Society 
These developments in interpolation theory have proved to be quite fruitful. The theorem of Riesz, as well as the classical theorem of Marcinkiewicz (see [7]), are consequences.

Many surprising and interesting relationships between well-known spaces such as $W^{s, p}, H^{s, p}$, and $B^{s, p}$ have been established by utilizing interpolation theory. See [7, p. 146] or [11, pp. 125-128].

Still another field in which complex interpolation theory has applications is elliptic partial differential equations. See [7], [13] and [14].

As one might suspect, in addition to usefulness in a variety of applications, the above-mentioned interpolation theories also have intrinsic mathematical elegance. A very comprehensive body of theory has been developed in each of the treatments referred to.

It is to this that we address this paper in large degree. The apparently diverse theories all have somewhat parallel developments. In particular, an "interpolation" theorem is of necessity proved in each. Elegant "duality" theorems appear in each.

In this paper we introduce the notion of "generalized interpolation space", and state and prove a "generalized interpolation theorem". This apparently provides a foundation for an axiomatic treatment of interpolation space theory, for we subsequently show that each of the aforementioned interpolation is a generalized interpolation space, and that each of the separate interpolation theorems is indeed a special case of the generalized interpolation theorem. Moreover, we use the generalized interpolation space concept to state and prove a generalized duality theorem. The very elegant duality theorems proved by Calderón, Lions-Peetre and Schechter, respectively, are shown to be special cases of our generalized duality theorem. Of special interest here is the isolation by the general theorem of the need in each of the separate theorems for certain "base" spaces to be duals of others.

Of special significance is the fact that, with our concept of generalized interpolation space, one is able to replace the ambiguous notion of "by the same construction" appearing in the standard definition of interpolation space (see $[6, p .5])$ with more substantive statements about the existence and commutation of certain linear operators.

It is of further significance that our generalized interpolation space concept and our generalized interpolation theorem structure provide an effective and practical tool for constructing interpolation spaces.

In this paper we provide four examples of such constructions.

II. The generalized interpolation theorem. In this section we state and prove a generalized interpolation theorem for Banach spaces. This result yields a simple but useful corollary.

As a consequence of the generalized interpolation theorem, we make a natural definition of generalized interpolation space. Subsequently, we show that the 
interpolation constructions of Calderón, Lions-Peetre and Schechter are all examples of generalized interpolation spaces. Furthermore, the interpolation theorems proved by the above mentioned mathematicians are shown to be special cases of the generalized interpolation theorem.

Finally we employ our generalized interpolation theorem construction to create new classes of interpolation spaces which enjoy a certain relationship with the mean spaces of Lions-Peetre.

Theorem 2.1 (Generalized Interpolation TheOrem). Suppose

(1) $X, Y, C$, and $D$ are Banach spaces,

(2) $T_{1}$ is a bounded linear operator from $C$ to $X$, and $T_{2}$ is a bounded linear operator from $D$ to $Y$,

(3) $L$ is a linear mapping of $X$ to $Y$ which induces a bounded linear mapping $L^{\prime}$ of $C$ to $D$ satisfying

$$
\begin{array}{rl}
L T_{1}=T_{2} L^{\prime} & \text { (see diagram below). } \\
X \stackrel{L}{X} Y & Y \\
T_{1} \uparrow_{C \stackrel{L^{\prime}}{\longrightarrow}}^{\longrightarrow} T_{2}
\end{array}
$$

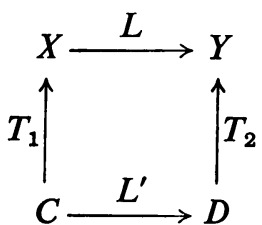

Then (1) $T_{1}(C)=\left\{T_{1}(f) \mid f \in C\right\}$ is a Banach space under the norm

$$
\|x\|_{T_{1}(C)}=\inf _{f \in C}\left\{\|f\|_{C} \mid T_{1}(f)=x\right\} .
$$

(2) $T_{2}(D)=\left\{T_{2}(g) \mid g \in D\right\}$ is a Banach space under the norm

$$
\|y\|_{T_{2}(D)}=\inf _{g \in D}\left\{\|g\|_{D} \mid T_{2}(g)=y\right\} .
$$

(3) $L$ is a bounded linear operator from $T_{1}(C)$ to $T_{2}(D)$, with $\|L\| \leqq\left\|L^{\prime}\right\|$.

Proof. (1) $T_{1}(C)$ is a Banach space under the above norm since it is isomorphic and isometric to the quotient space $C / N_{T_{1}}$, where $N_{T_{1}}$ is the kernel of $T_{1}$.

(2) Similarly, one shows that $T_{2}(D)$ is a Banach space.

(3) Let $x \in T_{1}(C)$. Then there exists $f \in C$ such that $T_{1} f=x$. Thus $L x=L T_{1} f$ $=T_{2} L^{\prime} f$. Hence $L x \in T_{2}(D)$ and $L$ maps $T_{1}(C)$ into $T_{2}(D)$. $L$ was given linear on $X$ and therefore is also linear on $T_{1}(C)$. Moreover, $L$ is bounded on $T_{1}(C)$ for let $x \in T_{1}(C)$. Let $\varepsilon>0$ be given. Then there exists $f \in C$ such that $\|x\|_{T_{1}(C)}+\varepsilon>\|f\|_{C}$ and $T_{1} f=x$. Thus

$$
\begin{aligned}
\|L x\|_{T_{2}(D)} & =\left\|L T_{1} f\right\|_{T_{2}(D)}=\left\|T_{2} L^{\prime} f\right\|_{T_{2}(D)} \\
& \leqq\left\|L^{\prime} f\right\|_{D} \leqq\left\|L^{\prime}\right\|\|f\|_{C} \leqq\left\|L^{\prime}\right\|\left(\|x\|_{C}+\varepsilon\right) .
\end{aligned}
$$

Corollary. Let $C, X$ be Banach spaces. Let $T$ be a bounded linear operator from $C$ to $X$. Suppose a linear mapping $L$ of $X$ into itself induces a bounded linear mapping 
$L^{\prime}$ of $C$ into itself which satisfies $L T=T L^{\prime}$. Then $L$ is a bounded linear mapping of $T(C)$ (with quotient space norm defined in (1) above) into itself.

Proof. An immediate consequence of Theorem 2.1.

DefinItION. Let a Banach space $X$ be given. Let $T$ be a bounded linear operator from a Banach space $C$ to $X$. Then the Banach space $T(C)$ under the norm

$$
\|x\|_{T(C)}=\inf _{f \in C}\left\{\|f\|_{C} \mid T(f)=x\right\}
$$

is said to be generalized interpolation space in $X$ (relative to $T$ and $C$ ).

In justification of our designation of Theorem 2.1 as the generalized interpolation theorem, we show in the sequel that the complex interpolation spaces $\left(X_{0}, X_{1}\right)_{T}$ and $\left(X_{0}, X_{1}\right)^{T}$ of Schechter, the complex interpolation space $\left(X_{0}, X_{1}\right)_{s}$ of Calderón, and the mean interpolation space (espace de moyennes) $S\left(p_{0}, r_{0}, X_{0} ; p_{1}, r_{1}, X_{1}\right)$, of Lions-Peetre are all generalized interpolation spaces. In particular, the respective interpolation theorems established separately for these spaces are all special cases of the generalized interpolation theorem.

We recall now the definition of $\left(X_{0}, X_{1}\right)_{s}$, the Calderón interpolation space. See [2, p. 114]. Let $X_{0}, X_{1}$ be Banach spaces with continuous embeddings in a topological vector space $V$. Then

(1) $X_{0}+X_{1}$ with norm defined by

$$
\|x\|_{x_{0}+x_{1}}=\inf _{x_{0}+x_{1}=x}\left\{\left\|x_{0}\right\|_{x_{0}}+\left\|x_{1}\right\|_{x_{1}}\right\}
$$

is a Banach space. See [2, p. 128].

Let $S$ be the open strip between 0 and 1 in the complex plane, that is, the set of complex numbers with real part greater than 0 and less than 1 . Let $H\left(X_{0}, X_{1}\right)$ be the set of all functions $f$ from the closure of $S$ to $X_{0}+X_{1}$ which satisfy:

(i) $f$ is analytic in $S$,

(ii) $f$ is continuous and bounded on the closure of $S$,

(iii) for $j=0,1$, there exist constants $K_{j}$ such that $\|f(j+i t)\|_{X_{j}} \leqq K$, for all real $t$. Then

(2) $H\left(X_{0}, X_{1}\right)$ is a Banach space under the norm

$$
\|f\|_{H\left(X_{0}, X_{1}\right)}=\max _{j=0,1}\left\{\sup _{t}\|f(j+i t)\|_{X_{j}}\right\} \quad[2, \text { p. 129]. }
$$

Let $s$ satisfying $0 \leqq s \leqq 1$ be fixed. Then

(3) $\left(X_{0}, X_{1}\right)_{s}=\left\{x \mid x=f(s), f \in H\left(X_{0}, X_{1}\right)\right\}$ is a Banach space under the norm

$$
\|x\|_{\left(x_{0}, x_{1}\right)}=\inf _{f(s)=x}\left\{\|f\|_{H\left(X_{0}, x_{1}\right)}\right\} \quad[2, \text { p. 129]. }
$$

(4) Let the mapping $T$ of $H\left(X_{0}, X_{1}\right)$ into $X_{0}+X_{1}$ be defined by $T(f)=f(s)$ for each $f$ belonging to $H\left(X_{0}, X_{1}\right)$.

THEOREM 2.2. $\left(X_{0}, X_{1}\right)_{s}$ is a generalized interpolation space in $X_{0}+X_{1}$ (relative to $T$ and $\left.H\left(X_{0}, X_{1}\right)\right)$. 
Proof. In view of (2), (3) and (4) above, it is sufficient to show that $T$ is a bounded linear mapping from $H\left(X_{0}, X_{1}\right)$ to $X_{0}+X_{1}$. Let $f, g$ be members of $H\left(X_{0}, X_{1}\right)$, and $c, d$ complex numbers. Then $T(c f+d g)=c f(s)+d g(s)=c T(f)+d T(g)$. Thus, $T$ is a linear operator.

For each $f$ belonging to $H\left(X_{0}, X_{1}\right)$,

$$
\begin{aligned}
\|T(f)\|_{X_{0}+X_{1}} & =\|f(s)\|_{X_{0}+X_{1}} \\
& \leqq \max _{j=0,1}\left\{\sup _{t}\|f(j+i t)\|_{X_{0}+X_{1}}\right\}
\end{aligned}
$$

(by the maximum modulus theorem for analytic functions defined in the strip; see [16])

$$
\leqq \max _{j=0,1}\left\{\sup _{t}\|f(j+i t)\|_{x_{j}}\right\}
$$

(by the definition of norm in $X_{0}+X_{1}$ )

$$
=\|f\|_{H\left(X_{0}, X_{1}\right)} \text {. }
$$

Consequently, $T$ is a bounded operator from $H\left(X_{0}, X_{1}\right)$ to $X_{0}+X_{1}$ with norm less than or equal to 1 .

We proceed now to state the Calderón interpolation theorem, and to prove it by use of its generalized interpolation space structure exhibited above and the generalized interpolation theorem, Theorem 2.1.

CALderón INTERPolation TheOREM. Let $L$ be a linear mapping from $X_{0}+X_{1}$ to $Y_{0}+Y_{1}$ which satisfies the following:

(1) The restriction of $L$ to $X_{0}$ is a bounded linear operator from $X_{0}$ to $Y_{0}$.

(2) The restriction of $L$ to $X_{1}$ is a bounded linear operator from $X_{1}$ to $Y_{1}$.

Then the restriction of $L$ to $\left(X_{0}, X_{1}\right)_{s}$ is a bounded linear operator from $\left(X_{0}, X_{1}\right)_{s}$ to $\left(Y_{0}, Y_{1}\right)_{s}$.

Proof. It will suffice to establish that $L$ induces a bounded linear mapping $L^{\prime}$ of $H\left(X_{0}, X_{1}\right)$ into $H\left(Y_{0}, Y_{1}\right)$ which satisfies $L T_{1}=T_{2} L^{\prime}$, where $T_{1}$ is defined by $T_{1}(f)=f(s)$ for each $f$ in $H\left(X_{0}, X_{1}\right)$, and $T_{2}$ is defined by $T_{2}(g)=g(s)$ for each $g$ in $H\left(Y_{0}, Y_{1}\right)$.

$T_{1}$ and $T_{2}$ are bounded linear operators as shown in the proof of Theorem 2.2.

Let a mapping $L^{\prime}$ on $H\left(X_{0}, X_{1}\right)$ be defined by $L^{\prime}(f)=L f$ where $L f$ is a composite function defined on the closure of the open strip $S$ in the complex plane with range values in $Y_{0}+Y_{1}$. That is, $(L f) z=L(f(z))$ for each $z$ with real part greater than or equal to 0 and less than or equal to 1 .

To show that $L^{\prime}$ is well defined and is a bounded linear operator from $H\left(X_{0}, X_{1}\right)$ to $H\left(Y_{0}, Y_{1}\right)$, we establish first that the given linear mapping $L$ from $X_{0}+X_{1}$ to $Y_{0}+Y_{1}$ is bounded. By assumption (1), $L$ (its restriction) is a bounded linear operator from $X_{0}$ to $Y_{0}$. We denote its norm by $M$. Similarly, by assumption (2), $L$ is a bounded linear operator from $X_{1}$ to $Y_{1}$. We denote its norm by $N$. Now, let 
$x$ be a member of $X_{0}+X_{1}$. Let $x_{0}, x_{1}$ be an arbitrary pair satisfying: $x_{0}$ is a member of $X_{0}, x_{1}$ is a member of $X_{1}$, and $x_{0}+x_{1}=x$. Then

$$
\begin{aligned}
\|L x\|_{Y_{0}+Y_{1}} & =\left\|L\left(x_{0}+x_{1}\right)\right\|_{Y_{0}+Y_{1}} \\
& \leqq\left\|L x_{0}+L x_{1}\right\|_{Y_{0}+Y_{1}} \leqq\left\|L x_{0}\right\|_{Y_{0}}+\left\|L x_{1}\right\|_{Y_{1}} \\
& \leqq M\left\|x_{0}\right\|_{X_{0}}+N\left\|x_{1}\right\|_{X_{1}} \leqq \max (M, N)\left(\left\|x_{0}\right\|_{X_{0}}+\left\|x_{1}\right\|_{X_{1}}\right) .
\end{aligned}
$$

Since $x_{0}, x_{1}$ is an arbitrary pair satisfying: $x_{0}$ is a member of $X_{0}, x_{1}$ is a member of $X_{1}$, and $x_{0}+x_{1}=x$,

$$
\begin{aligned}
\|L x\|_{Y_{0}+Y_{1}} & \leqq \max (M, N) \inf _{X_{0}+X_{1}=X}\left\{\left(\left\|x_{0}\right\|_{X_{0}}+\left\|x_{1}\right\|_{X_{1}}\right)\right\} \\
& =\max (M, N)\|x\|_{X_{0}+X_{1}} .
\end{aligned}
$$

Thus $L$ is bounded on $X_{0}+X_{1}$ with norm less than or equal to $\max (M, N)$.

We now show that for $f$ belonging to $H\left(X_{0}, X_{1}\right), L^{\prime}(f)=L f$ is a member of $H\left(Y_{0}, Y_{1}\right)$.

(1) Let $z, w$ belong to the closure of $S$ in the complex plane. Then

$$
\begin{aligned}
\|L f(z)-L f(w)\|_{Y_{0}+Y_{1}} & =\|L(f(z)-f(w))\|_{Y_{0}+Y_{1}} \\
& \leqq\|L\|\|f(z)-f(w)\|_{X_{0}+X_{1}} .
\end{aligned}
$$

Hence the continuity of $L f$ is a consequence of the continuity of $f$ as a member of $H\left(X_{0}, X_{1}\right)$, and the continuity of $L$ as a mapping of $X_{0}+X_{1}$ into $Y_{0}+Y_{1}$.

(2) Similarly, for $z, w$ in the open strip $S$,

$$
\begin{aligned}
\left\|(L f(w)-L f(z)) /(w-z)-L\left(f^{\prime}(z)\right)\right\|_{Y_{0}+Y_{1}} & =\left\|L\left((f(w)-f(z)) /(w-z)-f^{\prime}(z)\right)\right\|_{Y_{0}+Y_{1}} \\
& \leqq\|L\|\left\|(f(w)-f(z)) /(w-z)-f^{\prime}(z)\right\|_{X_{0}+X_{1}} .
\end{aligned}
$$

Thus the analyticity of $L f$ in the strip is a consequence of the analyticity of $f$ and the continuity of $L$.

(3) Recall that the norm of $L$ mapping $X_{0}$ into $Y_{0}$ is $M$, and the norm of $L$ mapping $X_{1}$ into $Y_{1}$ is $N$. Thus for $j=0,1$,

$$
\|L f(j+i t)\|_{Y_{j}} \leqq \max (M, N)\|f(j+i t)\|_{X_{j}} .
$$

Hence the boundedness of $f$ on the edges of the strip $S$ implies that $L f$ is also bounded on said edges.

(1), (2) and (3) above clearly imply that for $f$ a member of $H\left(X_{0}, X_{1}\right), L f=L^{\prime}(f)$ is a member of $H\left(Y_{0}, Y_{1}\right)$. So $L^{\prime}$ is indeed a mapping from $H\left(X_{0}, X_{1}\right)$ to $H\left(Y_{0}, Y_{1}\right)$. Let $f, g$ be members of $H\left(X_{0}, X_{1}\right)$, and $c, d$ complex numbers. Then

$$
\begin{aligned}
L^{\prime}(c f+d g) & =L(c f+d g) \\
& =L(c f)+L(d g)=c(L f)+d(L g) \\
& =c L^{\prime}(f)+d L^{\prime}(g) .
\end{aligned}
$$


Thus $L^{\prime}$ is a linear operator. Furthermore, $L^{\prime}$ is bounded, for condition (3) above yields

$$
\begin{aligned}
\left\|L^{\prime}(f)\right\|_{H\left(Y_{0}, Y_{1}\right)} & =\max _{j=0,1}\left\{\sup _{t}\|L f(j+i t)\|_{Y_{j}}\right\} \\
& \leqq \max _{j=0,1}\left\{\sup _{t} \max (M, N)\|f(j+i t)\|_{X_{j}}\right\} \\
& =\max (M, N)\left(\max _{j=0,1}\left\{\sup _{t}\|f(j+i t)\|_{X_{j}}\right\}\right) \\
& =\max (M, N)\|f\|_{H\left(X_{0}, X_{1}\right)} .
\end{aligned}
$$

Observe finally, that for each $f$ in $H\left(X_{0}, X_{1}\right)$,

$$
L T_{1}(f)=L(f(s))=L f(s)=L^{\prime}(f)(s)=T_{2}\left(L^{\prime}(f)\right)=T_{2} L^{\prime}(f) .
$$

Thus, $L T_{1}=T_{2} L^{\prime}$.

With this result, the hypotheses of the generalized interpolation theorem are all satisfied, and the conclusion of the Calderón interpolation theorem follows.

We give now the definition of $S\left(p_{0}, r_{0}, X_{0} ; p_{1}, r_{1}, X_{1}\right)$, the Lions-Peetre "mean" interpolation space. As before, let $X_{0}, X_{1}$ be Banach spaces with continuous embeddings in a topological vector space $V$. Let the Banach space $X_{0}+X_{1}$ be defined as before. Let $p_{0}, p_{1}$ be real numbers which are greater than or equal to 1 , and less than or equal to "infinity". Let $r_{0}, r_{1}$ be real numbers satisfying $r_{0} r_{1}$ is less than 0 (say $r_{0}$ is positive and $r_{1}$ is negative). Let $W=W\left(p_{0}, r_{0}, X_{0} ; p_{1}, r_{1}, X_{1}\right)$ be the set of all functions $f$ with domain $R$, the set of reals, and range values in $X_{0}+X_{1}$ which satisfy the following:

(1) $e^{r_{0} t} f$ is a member of $L^{p_{0}}\left(X_{0}\right)$,

(2) $e^{r_{1} t} f$ is a member of $L^{p_{1}}\left(X_{1}\right)$.

$W$ is a Banach space under the norm

$$
\|f\|_{w}=\max \left\{\left\|e^{r_{0} t} f\right\|_{L^{p_{0}\left(X_{0}\right)}},\left\|e^{r_{1} t} f\right\|_{L^{p_{1}\left(X_{1}\right)}}\right\} .
$$

See $[6$, p. 9].

For each $f$ in $W, \int_{-\infty}^{\infty} f(t) d t$ exists [6, p. 9].

Let $S=S\left(p_{0}, r_{0}, X_{0} ; p_{1}, r_{1}, X_{1}\right)$ be the set of all $x$ in $X_{0}+X_{1}$ such that $x=\int_{-\infty}^{\infty} f(t) d t$ where $f$ is a member of $W$. Under the norm

$$
\|x\|_{S}=\inf _{f \in W}\left\{\|f\|_{w} \mid x=\int_{-\infty}^{\infty} f(t) d t\right\}
$$

$S$ is a Banach space. See [6, p. 9].

Let the mapping $T$ of $W$ into $X_{0}+X_{1}$ be defined by

$$
T(f)=\int_{-\infty}^{\infty} f(t) d t
$$

THEOREM 2.3. $S$ is a generalized interpolation space in $X_{0}+X_{1}$ (relative to $T$ and $W$ ).

Proof. In view of the above definitions and remarks, it will suffice to show that 
$T$ is a bounded linear mapping from $W$ to $X_{0}+X_{1}$. Let $f, g$ be members of $W$, and $c, d$ complex numbers. Then

$$
\begin{aligned}
T(c f+d g) & =\int_{-\infty}^{\infty}(c f+d g) d t \\
& =c \int_{-\infty}^{\infty} f(t) d t+d \int_{-\infty}^{\infty} g(t) d t=c T(f)+d T(g)
\end{aligned}
$$

Thus, $T$ is linear. We proceed to show that $T$ is bounded. Let $q_{0}, q_{1}$ satisfy $1 / q_{0}+1 / p_{0}$ $=1=1 / q_{1}+1 / p_{1}$. Recall that $r_{0}$ is positive and $r_{1}$ is negative. Then

$$
\begin{aligned}
& \|T f\|_{x_{0}+x_{1}}=\left\|\int_{-\infty}^{\infty} f(t) d t\right\|_{x_{0}+x_{1}} \\
& \leqq \int_{-\infty}^{\infty}\|f(t)\|_{x_{0}+X_{1}} d t \\
& =\int_{0}^{\infty}\|f(t)\|_{X_{0}+X_{1}} d t+\int_{-\infty}^{0}\|f(t)\|_{X_{0}+x_{1}} d t \\
& =\int_{0}^{\infty}\left|e^{-r_{0} t}\right|\left\|e^{r_{0} t} f(t)\right\|_{X_{0}+X_{1}} d t+\int_{-\infty}^{0}\left|e^{-r_{1} t}\right|\left\|e^{r_{1} t} f(t)\right\|_{X_{0}+X_{1}} d t \\
& \leqq \int_{0}^{\infty}\left|e^{-r_{0} t}\right|\left\|e^{r_{0} t} f(t)\right\|_{x_{0}} d t \\
& +\int_{-\infty}^{0}\left|e^{-r_{1} t}\right|\left\|e^{r_{1} t} f(t)\right\|_{X_{1}} d t \quad \text { (definition of norm in } X_{0}+X_{1} \text { ) } \\
& =\left(\int_{0}^{\infty} \mid e^{-r_{0} t \mid q_{0}}\right)^{1 / q_{0}}\left(\int_{0}^{\infty}\left\|e^{r_{0} t} f(t)\right\|_{X_{0}}^{p_{0}}\right)^{1 / p_{0}} \\
& +\left(\int_{-\infty}^{0} \mid e^{-r_{1} t \mid q_{1}}\right)^{1 / q_{1}}\left(\int_{-\infty}^{0}\left\|e^{r_{1} t} f(t)\right\|_{X_{1}}^{p_{1}}\right)^{1 / p_{1}}
\end{aligned}
$$

(by assumed property of $f$ and Hölder's inequality)

$$
\begin{aligned}
\leqq & \left(\int_{0}^{\infty}\left|e^{-r_{0} t}\right|^{q_{0}}\right)^{1 / q_{0}}\left(\int_{-\infty}^{\infty}\left\|e^{r_{0} t} f(t)\right\|_{X_{0}}^{p_{0}}\right)^{1 / p_{0}} \\
& +\left(\int_{-\infty}^{0}\left|e^{-r_{1} t}\right|^{q_{1}}\right)^{1 / q_{1}}\left(\int_{-\infty}^{\infty}\left\|e^{r_{1} t} f(t)\right\|_{X_{1}}^{p_{1}}\right)^{1 / p_{1}} \\
\leqq & \left(\int_{0}^{\infty}\left|e^{-r_{0} t}\right|_{0}\right)^{1 / q_{0}}\left(\|f\|_{W}\right)+\left(\int_{-\infty}^{0}\left|e^{-r_{1} t}\right|^{q_{1}}\right)^{1 / q_{1}}\left(\|f\|_{W}\right) \\
= & \text { (constant) }\left(\|f\|_{W}\right) .
\end{aligned}
$$

Thus, the continuity of $T$ is clearly established.

We give now a statement of the Lions-Peetre interpolation theorem. As was the case with the Calderón interpolation theorem, we will utilize the generalized space structure together with the generalized interpolation theorem to provide a proof.

Lions-PeEtre Interpolation TheORem Let $L$ be a linear mapping from $X_{0}+X_{1}$ to $Y_{0}+Y_{1}$ which satisfies the following: 
(1) The restriction of $L$ to $X_{0}$ is a bounded linear operator from $X_{0}$ to $Y_{0}$ with norm $M$.

(2) The restriction of $L$ to $X_{1}$ is a bounded linear operator from $X_{1}$ to $Y_{1}$ with norm $N$.

Then the restriction of $L$ to $S\left(p_{0}, r_{0}, X_{0} ; p_{1}, r_{1}, X_{1}\right)$ is a bounded linear operator from $S_{X}=S\left(p_{0}, r_{0}, X_{0} ; p_{1}, r_{1}, X_{1}\right)$ to $S_{Y}=S\left(p_{0}, r_{0}, Y_{0} ; p_{1}, r_{1}, Y_{1}\right)$.

Proof. It is sufficient to show that $L$ induces a bounded linear mapping $L^{\prime}$ of $W_{X}=W\left(p_{0}, r_{0}, X_{0} ; p_{1}, r_{1}, X_{1}\right)$ into $W_{Y}=W\left(p_{0}, r_{0}, Y_{0} ; p_{1}, r_{1}, Y_{1}\right)$ which satisfies $L T_{1}=T_{2} L^{\prime}$, where $T_{1}$ is a mapping of $W_{X}$ into $X_{0}+X_{1}$ defined by

$$
T_{1}(f)=\int_{-\infty}^{\infty} f(t) d t
$$

and $T_{2}$ is a mapping of $W_{Y}$ into $Y_{0}+Y_{1}$ defined by

$$
T_{2}(g)=\int_{-\infty}^{\infty} g(t) d t
$$

$T_{1}$ and $T_{2}$ are bounded linear operators as shown in the proof of Theorem 2.3.

Let a mapping $L^{\prime}$ on $W_{X}$ be defined by $L^{\prime}(f)=L f$, where $L f(t)=L(f(t))$ for each real number $t$.

We now show that for $f$ belonging to $W_{X}, L^{\prime}(f)=L f$ is a member of $W_{Y}$. For each real number $t$, and for $i=0,1$,

$$
e^{r_{i} t} L(f(t))=L\left(e^{r_{i} t} f(t)\right) \quad(\text { since } L \text { is linear }) .
$$

Thus,

$$
\left\|e^{r_{i} t} L f\right\|_{Y_{i}} \leqq K_{i}\left\|e^{r_{i} t} f\right\|_{X_{i}} \quad\left(K_{i} \text { is the norm of } L \text { on } X_{i}\right) .
$$

Therefore $e^{r_{i} t} L f$ is a member of $L^{p_{i}}\left(Y_{i}\right)$ since $e^{r_{i} t} f$ is a member of $L^{p_{i}}\left(X_{i}\right)$. Hence, $L f$ is a member of $W_{Y}$.

Moreover, it is a consequence of the above inequalities that

$$
\|L f\|_{W_{Y}} \leqq \max (M, N)\|f\|_{W_{X}} \quad\left(\text { recall } K_{0}=M, K_{1}=N\right) .
$$

Thus $L^{\prime}$ is indeed a bounded operator from $W_{X}$ to $W_{Y}$. The linearity of $L^{\prime}$ is an immediate consequence of the linearity of $L$.

We show now that $L T_{1}=T_{2} L^{\prime}$. Recall from the proof of the Calderón interpolation theorem that $L$ is a bounded linear mapping from $X_{0}+X_{1}$ to $Y_{0}+Y_{1}$. Let $f$ be a member of $W_{X}$. Then

$$
\begin{aligned}
L T_{1}(f) & =L\left(T_{1}(f)\right)=L\left(\int_{-\infty}^{\infty} f(t) d t\right) \\
& =\int_{-\infty}^{\infty} L(f(t)) d t \quad \text { (since } L \text { is bounded) } \\
& =\int_{-\infty}^{\infty} L f(t) d t=T_{2}(L f)=T_{2} L^{\prime}(f) .
\end{aligned}
$$

Thus, $L T_{1}=T_{2} L^{\prime}$. 
We observe now that the conclusion of the Lions-Peetre interpolation theorem follows as a consequence of the generalized interpolation theorem.

We look now at the Schechter interpolation spaces and theorems. Let $X_{0}, X_{1}$, $X_{0}+X_{1}$ and $H\left(X_{0}, X_{1}\right)$ all be defined as before. Let $T$ be a two-dimensional distribution with compact support in the open strip $S$, and range values in $X_{0}+X_{1}$. Then

$$
\left(X_{0}, X_{1}\right)_{T}=\left\{T(f) \mid f \in H\left(X_{0}, X_{1}\right)\right\}
$$

is a Banach space under the norm

$$
\|x\|_{\left(x_{0}, X_{1}\right)_{T}}=\inf \left\{\|f\|_{H\left(X_{0}, X_{1}\right)} \mid f \in H\left(X_{0}, X_{1}\right), T(f)=x\right\} .
$$

See $[11$, p. 133].

THEOREM 2.4. $\left(X_{0}, X_{1}\right)_{T}$ is a generalized interpolation space in $X_{0}+X_{1}$ (relative to $T$ and $\left.H\left(X_{0}, X_{1}\right)\right)$.

Proof. One has only to show that $T$ is a bounded linear operator from $H\left(X_{0}, X_{1}\right)$ to $X_{0}+X_{1} . T$ is linear by its definition as a distribution. Furthermore, since $T$ has compact support in the open strip, there exists a constant $K$ such that

$$
\|T(f)\|_{X_{0}+X_{1}}=K\|f\|_{H\left(X_{0}, X_{1}\right)} \quad[11, \text { p. 133]. }
$$

Thus, the continuity of $T$ is clear.

SCHECHTER INTERPOLATION THEOREM (FOR $\left.\left(X_{0}, X_{1}\right)_{T}\right)$. Let $L$ be a linear mapping from $X_{0}+X_{1}$ to $Y_{0}+Y_{1}$ which satisfies the following:

(1) The restriction of $L$ to $X_{0}$ is a bounded linear operator from $X_{0}$ to $Y_{0}$ with norm $M$.

(2) The restriction of $L$ to $X_{1}$ is a bounded linear operator from $X_{1}$ to $Y_{1}$ with norm $N$.

Then the restriction of $L$ to $\left(X_{0}, X_{1}\right)_{T}$ is a bounded linear operator from $\left(X_{0}, X_{1}\right)_{T}$ to $\left(Y_{0}, Y_{1}\right)_{T}$.

Proof. As before it is sufficient to show that $L$ induces a bounded linear mapping $L^{\prime}$ of $H\left(X_{0}, X_{1}\right)$ into $H\left(Y_{0}, Y_{1}\right)$ which satisfies $L T_{1}=T_{2} L^{\prime}$ where both $T_{1}$ and $T_{2}$ are identified with a two-dimensional distribution $T$ with compact support in the open strip between 0 and 1 .

As in the proof of the Calderón interpolation theorem, let $L^{\prime}$ mapping $H\left(X_{0}, X_{1}\right)$ into $H\left(Y_{0}, Y_{1}\right)$ be defined by $L^{\prime}(f)=L f$.

Then, as shown previously, $L^{\prime}$ is a bounded linear operator from $H\left(X_{0}, X_{1}\right)$ to $H\left(Y_{0}, Y_{1}\right)$. Furthermore, it is a property of the distribution $T$ that $L T=T L^{\prime}$ [11, p. 135]. Thus, $L T_{1}=T_{2} L^{\prime}$.

Hence, the conclusion of the Schechter interpolation theorem for $\left(X_{0}, X_{1}\right)_{T}$ follows as a consequence of the generalized interpolation theorem. 
REMARK. The Calderón interpolation space and interpolation theorem are special cases of the above Schechter interpolation space and theorem, respectively, when $T$ is the delta functional.

We consider now a second interpolation space and interpolation theorem by Schechter. Let $C$ be the set of complex numbers. Let $T$ be a distribution as before other than the 0-distribution on $H(C, C)$. Let $S\left(X_{0}, X_{1}\right)$ be the set of all members $f$ in $H\left(X_{0}, X_{1}\right)$ for which there exists $x$ in $X_{0}+X_{1}$ satisfying

$$
f T=x T \text { (in the sense of distributions). }
$$

That is, for $\phi$ belonging to $H(C, C), T(f \phi)=x T(\phi) . S\left(X_{0}, X_{1}\right)$ is a closed subspace of $H\left(X_{0}, X_{1}\right)$ [11, pp. 133-134]. Thus $S\left(X_{0}, X_{1}\right)$ is a Banach subspace of $H\left(X_{0}, X_{1}\right)$.

Let the mapping $T$ of $S\left(X_{0}, X_{1}\right)$ into $X_{0}+X_{1}$ be defined by $T(f)=x$, where $x$ satisfies $f T=x T$.

This mapping is well defined, for suppose $x_{1} T=x_{2} T$. Then by definition for each $\phi$ in $H(C, C), x_{1} T(\phi)=x_{2} T(\phi)$. However, $T$ by assumption is nontrivial on $H(C, C)$. Therefore there exists $\phi$ in $H(C, C)$ such that $T(\phi) \neq 0$. Hence, $x_{1}=x_{2}$.

Let $\left(X_{0}, X_{1}\right)^{T}=T\left(S\left(X_{0}, X_{1}\right)\right)$, the image set in $X_{0}+X_{1}$ of $S\left(X_{0}, X_{1}\right)$ under $T$. Then $\left(X_{0}, X_{1}\right)^{T}$ is a Banach space under the norm

See [11, pp. 133-134].

$$
\|x\|_{\left(X_{0}, X_{1}\right)^{T}}=\inf _{f \in S\left(X_{0}, X_{1}\right)}\left\{\|f\|_{S\left(X_{0}, X_{1}\right)} \mid f t=x T\right\} .
$$

THEOREM 2.5. $\left(X_{0}, X_{1}\right)^{T}$ is a generalized interpolation space in $X_{0}+X_{1}$ (relative to $T$ and $S\left(X_{0}, X_{1}\right)$ ).

Proof. It is enough to show that $T$ is a bounded linear operator from $S\left(X_{0}, X_{1}\right)$ to $X_{0}+X_{1} . T$ is linear, for suppose $f, g$ are members of $S\left(X_{0}, X_{1}\right)$ and $c, d$ are complex numbers. Let $T(f)=x_{1}, T(g)=x_{2}$ and $T(c f+d g)=x$, then $f T=x_{1} T, g T=x_{2} T$ and $(c f+d g) T=x T$ by definition. Thus,

$$
\begin{aligned}
& x T=(c f+d g) T=(c f) T+(d g) T \quad \text { (property of distributions) } \\
& =c(f T)+d(g T) \quad, \quad, \quad, \\
& =c\left(x_{1} T\right)+d\left(x_{2} T\right) \quad, \quad, \quad, \quad, \\
& =\left(c x_{1}\right) T+\left(d x_{2}\right) T \quad, \quad,, \quad, \\
& =\left(c x_{1}+d x_{2}\right) T \quad,, \quad, \quad,
\end{aligned}
$$

Therefore, $x=c x_{1}+d x_{2}$, as shown in the above proof that $T$ is a well-defined mapping. Thus,

$$
T(c f+d g)=c T(f)+d T(g),
$$

and linearity is established. 
$T$ is a continuous mapping from $S\left(X_{0}, X_{1}\right)$ to $X_{0}+X_{1}$, for suppose that $f_{n}$ converges to $f$ in $S\left(X_{0}, X_{1}\right)$. Let $T\left(f_{n}\right)=x_{n}$, and $T(f)=x$. Let $\phi$ in $H(C, C)$ be such that $T(\phi) \neq 0$. Then observe that

$$
\begin{aligned}
\left\|x_{n}-x\right\|_{X_{0}+x_{1}}|T(\phi)| & =\left\|\left(x_{n} T\right) \phi-(x T) \phi\right\|_{x_{0}+x_{1}} \\
& =\left\|T\left(f_{n} \phi\right)-T(f \phi)\right\|_{x_{0}+x_{1}} \\
& =\left\|T\left(f_{n} \phi-f \phi\right)\right\|_{x_{0}+x_{1}} \\
& \leqq K\|\phi\|_{H(C, C)}\left\|f_{n}-f\right\|_{S\left(X_{0}, X_{1}\right)}
\end{aligned}
$$

(where $K$ is a constant depending only on $T$ since $T$ has compact support). See $\left[11\right.$, p. 133]. It is now clear that as $f_{n}$ converges to $f$ in $S\left(X_{0}, X_{1}\right),\left\|x_{n}-x\right\|_{X_{0}+X_{1}}$ converges to 0 . Thus, $x_{n}$ converges to $x$ in $X_{0}+X_{1}$. Hence, $T\left(f_{n}\right)$ converges to $T(f)$ in $X_{0}+X_{1}$, and the continuity of $T$ is clear.

SCHEChter INTERPolation THeOREM (FOR $\left.\left(X_{0}, X_{1}\right)^{T}\right)$. Let $L$ be a linear mapping from $X_{0}+X_{1}$ to $Y_{0}+Y_{1}$ which satisfies the following:

(1) The restriction of $L$ to $X_{0}$ is a bounded linear operator from $X_{0}$ to $Y_{0}$ with norm $M$.

(2) The restriction of $L$ to $X_{1}$ is a bounded linear operator from $X_{1}$ to $Y_{1}$ with norm $N$.

Then the restriction of $L$ to $\left(X_{0}, X_{1}\right)^{T}$ is a bounded linear operator from $\left(X_{0}, X_{1}\right)^{T}$ to $\left(Y_{0}, Y_{1}\right)^{T}$.

Proof. Once again it is sufficient to show that $L$ induces a bounded linear mapping $L^{\prime}$ of $S\left(X_{0}, X_{1}\right)$ into $S\left(Y_{0}, Y_{1}\right)$ which satisfies $L T_{1}=T_{2} L^{\prime}$ where both $T_{1}$ and $T_{2}$ are identified with a two-dimensional distribution $T$ of the type considered above.

Let a mapping $L^{\prime}$ on $S\left(X_{0}, X_{1}\right)$ be defined by $L^{\prime}(f)=L f$, where $L f$ is defined as in the Calderón and previous Schechter interpolation theorems. As has already been established in each of these theorems, $L f$ is a member of $H\left(Y_{0}, Y_{1}\right)$ and

$$
\|L f\|_{H\left(Y_{0}, Y_{1}\right)} \leqq \max (M, N)\|f\|_{H\left(X_{0}, X_{1}\right)} .
$$

Since $S\left(X_{0}, X_{1}\right), S\left(Y_{0}, Y_{1}\right)$ are respectively Banach subspaces of $H\left(X_{0}, X_{1}\right)$ and $H\left(Y_{0}, Y_{1}\right)$, and in the light of the above inequality, one establishes that $L^{\prime}$ is a bounded operator from $S\left(X_{0}, X_{1}\right)$ to $S\left(Y_{0}, Y_{1}\right)$ simply by showing that for each $f$ in $S\left(X_{0}, X_{1}\right), L^{\prime}(f)=L f$ is a member of $S\left(Y_{0}, Y_{1}\right)$. We see this as follows: Let $f$ be a member of $S\left(X_{0}, X_{1}\right)$. Then there exists $x$ in $X_{0}+X_{1}$ such that $f T=x T$. Now let $\phi$ be a member of $H(C, C)$. Then

$$
\begin{aligned}
(L f) T(\phi) & =T(L f) \phi=T(L(f \phi)) \\
& =L(T(f \phi))=L(x(T(\phi)))=(L x) T(\phi) .
\end{aligned}
$$

Thus, $(L f) T=(L x) T . L x$ is a member of $Y_{0}+Y_{1}$, and therefore $L f$ is in $S\left(Y_{0}, Y_{1}\right)$ as was to be shown. 
The linearity of $L^{\prime}$ follows directly as in the proof of the previous Schechter interpolation theorem.

We show now that $L T_{1}=T_{2} L^{\prime}$. Let $T_{1}(f)=x$ and $T_{2}(L f)=y$. Then it is sufficient to show that $L x=y$ for

$$
L x=L\left(T_{1}(f)\right)=\left(L T_{1}\right) f, \quad \text { and } \quad y=T_{2}(L f)=\left(T_{2} L^{\prime}\right) f
$$

Let $\phi$ be a member of $H(C, C)$ such that $T \phi \neq 0$. Then the equality $y=T_{2}(L f)$ implies

$$
\begin{aligned}
y(T(\phi)) & =T((L f) \phi)=T(L(f \phi)) \\
& =L(T(f \phi))=L(x(T(\phi))=(L x) T(\phi)) .
\end{aligned}
$$

Thus, $y=L x$, and we have the required commutativity of maps.

Hence, the conclusion of the second Schechter interpolation theorem follows as a consequence of the generalized interpolation theorem.

Lions and Peetre suggest that interpolation spaces other than the mean spaces, $S\left(p_{0}, r_{0}, X_{0} ; p_{1}, r_{1}, X_{1}\right)$, might be constructed by use of weights on functions other than $e^{r_{0} t}, e^{r_{1} t}$, occurring in $W\left(p_{0}, r_{0}, X_{0} ; p_{1}, r_{1}, X_{1}\right)$. See [6].

Our generalized interpolation theorem suggests an alternate method of constructing Banach spaces for which an interpolation theorem is valid, which involves producing appropriate bounded linear operators on $W\left(p_{0}, r_{0}, X_{0} ; p_{1}, r_{1}, X_{1}\right)$ other than the integral operator used by Lions-Peetre. We pursue this point of view in examples that follow. In particular, we seek operators $T_{1}, T_{2}$, such that

(1) $T_{1}$ is a bounded linear operator from $W_{X}=W\left(p_{0}, r_{0}, X_{0} ; p_{1}, r_{1}, X_{1}\right)$ to $X_{0}+X_{1}$, and $T_{2}$ is a bounded linear operator from $W_{Y}=W\left(p_{0}, r_{0}, Y_{0} ; p_{1}, r_{1}, Y_{1}\right)$ to $Y_{0}+Y_{1}$.

(2) $T_{1}=T_{2}$ if $X_{0}=Y_{0}$ and $X_{1}=Y_{1}$.

(3) $L T_{1}=T_{2} L^{\prime}$, where $L$ is any linear mapping of $X_{0}+X_{1}$ into $Y_{0}+Y_{1}$ such that

(i) the restriction of $L$ to $X_{0}$ is a bounded linear operator from $X_{0}$ to $Y_{0}$,

(ii) the restriction of $L$ to $X_{1}$ is a bounded linear operator from $X_{1}$ to $Y_{1}$, and $L^{\prime}$ is the bounded linear mapping from $W_{X}$ to $W_{Y}$, defined as before in the Lions-Peetre interpolation theorem by $L^{\prime}(f)=L f$.

EXAMPLE 1 . Let $m$ be a fixed positive integer. Let $T_{1}$ mapping $W_{X}$ into $X_{0}+X_{1}$ be defined by

$$
T_{1}(f)=\int_{-m}^{m} f(t) d t
$$

Let $T_{2}$ mapping $W_{Y}$ into $Y_{0}+Y_{1}$ be defined by

$$
T_{2}(f)=\int_{-m}^{m} f(t) d t
$$


We show now that conditions (1), (2) and (3) above are indeed satisfied.

$$
\begin{aligned}
\left\|T_{1}(f)\right\|_{X_{0}+X_{1}} & =\left\|\int_{-m}^{m} f(t) d t\right\|_{X_{0}+X_{1}} \\
& \leqq \int_{-m}^{m}\|f(t)\|_{X_{0}+X_{1}} d t \\
& \leqq \int_{-\infty}^{\infty}\|f(t)\|_{X_{0}+X_{1}} d t \\
& =K\|f\|_{W_{X}}
\end{aligned}
$$

(where $K$ is independent of $f$ as shown in the proof of the Lions-Peetre interpolation theorem). Thus, $T_{1}$ is a bounded operator. Its linearity is easily seen. Similarly, $T_{2}$ is shown to be a bounded linear operator.

It is clear too that $T_{1}=T_{2}$ if $X_{0}=Y_{0}$ and $X_{1}=Y_{1}$. Observe finally that

$$
\begin{aligned}
L T_{1}(f) & =L\left(\int_{-m}^{m} f(t) d t\right)=\int_{-m}^{m}(L f) t d t \\
& =T_{2}(L f)=T_{2}\left(L^{\prime}(f)\right)=\left(T_{2} L^{\prime}\right) f .
\end{aligned}
$$

Thus, $L T_{1}=T_{2} L^{\prime}$.

EXAMPLE 2. Let $h$ be any bounded, measurable and scalar-valued function on $R$, the set of reals. Define $T_{1}$ mapping $W_{X}$ into $X_{0}+X_{1}$ by

$$
T_{1}(f)=\int_{-\infty}^{\infty}(h f) d t
$$

Similarly, define $T_{2}$ mapping $W_{Y}$ into $Y_{0}+Y_{1}$ by

$$
T_{2}(f)=\int_{-\infty}^{\infty}(h f) d t .
$$

Let $M$ be a bound for $h$ on $R$. Then as before there exists a constant $K$ such that

$$
\left\|T_{1}(f)\right\|_{X_{0}+X_{1}} \leqq M K\|f\|_{W_{X}} .
$$

Thus, $T_{1}$ is bounded. Its linearity is clear. Similarly $T_{2}$ is bounded and linear. It is immediate too that $T_{1}=T_{2}$ whenever $X_{0}=Y_{0}$ and $X_{1}=Y_{1}$. Moreover, for $f$ in $W_{X}$,

$$
\begin{aligned}
& \left(L T_{1}\right) f=L\left(\int_{-\infty}^{\infty}(h f) d t\right)=\int_{-\infty}^{\infty} L(h f) d t \\
& =\int_{-\infty}^{\infty} h(L f) d t=T_{2}(L f)=T_{2}\left(L^{\prime}(f)\right)=\left(T_{2} L^{\prime}\right) f .
\end{aligned}
$$

Thus, $L T_{1}=T_{2} L^{\prime}$.

EXAMPLE 3. We assume in this example that the $p_{0}, p_{1}$ of the spaces $W_{X}, W_{Y}$ both equal infinity. $L^{p_{0}}\left(X_{0}\right)$ is here defined to be the set of bounded functions from $R$ to $X_{0} . L^{p_{1}}\left(X_{1}\right), L^{p_{0}}\left(Y_{0}\right)$ and $L^{p_{1}}\left(Y_{1}\right)$ are similarly defined. All of these spaces are normed with the usual supremum norms. 
Define $T_{1}$ mapping $W_{X}$ into $X_{0}+X_{1}$ by $T_{1}(f)=f(0)$. Similarly define $T_{2}$ mapping $W_{Y}$ into $Y_{0}+Y_{1}$ by $T_{2}(f)=f(0)$. Then,

$$
\begin{aligned}
\left\|T_{1}(f)\right\|_{X_{0}+X_{1}} & =\|f(0)\|_{x_{0}+x_{1}} \leqq\|f(0)\|_{x_{0}} \\
& \leqq \max _{j=0,1}\left\{\sup _{t}\left\|e^{r_{f} t} f(t)\right\|_{x_{j}}\right\}=\|f\|_{w_{X}} .
\end{aligned}
$$

Thus, $T_{1}$ is bounded. Similarly, one shows that $T_{2}$ is bounded. Linearity is clear. Further,

$$
\begin{aligned}
\left(L T_{1}\right) f & =L\left(T_{1}(f)\right)=L(f(0))=(L f)(0) \\
& =T_{2}(L f)=T_{2}\left(L^{\prime}(f)\right)=\left(T_{2} L^{\prime}\right) f
\end{aligned}
$$

Thus $L T_{1}=T_{2} L^{\prime}$. It is evident too that $T_{1}=T_{2}$ if $X_{0}=Y_{0}$ and $X_{1}=Y_{1}$.

EXAMPLE 4. Let $p_{0}, p_{1}$, and related spaces be defined as in Example 3 above. Define $T_{1}$ mapping $W_{X}$ into $X_{0}+X_{1}$ by

$$
T_{1}(f)=\sum_{-\infty}^{\infty} f(n)
$$

Similarly, define $T_{2}$ mapping $W_{Y}$ into $Y_{0}+Y_{1}$ by

$$
T_{2}(f)=\sum_{-\infty}^{\infty} f(n)
$$

If $f$ is a member of $W_{X}$, for $j=0,1, e^{r_{f} t} f(t)$ is a member of $L^{p_{j}}\left(X_{j}\right)$. In which case, $\left\|e^{r}, t f(t)\right\|_{x_{j}}$ is bounded for $j=0,1$. Let

$$
B_{j}=\sup _{t}\left\{\left\|e^{r}, t f(t)\right\|_{x_{j}}\right\}, \quad j=0,1 .
$$

Then for each integer $n$,

$$
\|f(n)\|_{X_{j}} \leqq B_{j} e^{-n r_{j}}, \quad j=0,1 .
$$

We proceed now to show that $T_{1}$ is a bounded mapping from $W_{X}$ to $X_{0}+X_{1}$. Recall that $r_{0}$ is a positive real number and $r_{1}$ is negative.

$$
\begin{aligned}
\left\|T_{1}(f)\right\|_{x_{0}+x_{1}} & =\left\|\sum_{-\infty}^{\infty} f(n)\right\|_{x_{0}+x_{1}} \\
& =\left\|\sum_{0}^{\infty} f(n)+\sum_{-\infty}^{-1} f(n)\right\|_{x_{0}+x_{1}} \\
& \leqq \sum_{0}^{\infty}\|f(n)\|_{x_{0}+x_{1}}+\sum_{-\infty}^{-1}\|f(n)\|_{x_{0}+x_{1}} \\
& \leqq \sum_{0}^{\infty}\|f(n)\|_{x_{0}}+\sum_{-\infty}^{-1}\|f(n)\|_{x_{1}} \\
& =\sum_{0}^{\infty}\|f(n)\|_{x_{0}}+\sum_{1}^{\infty}\|f(-n)\|_{X_{1}} \\
& \leqq \sum_{0}^{\infty} B_{0} e^{-n r_{0}}+\sum_{1}^{\infty} B_{1} e^{n r_{1}} \\
& =B_{0}\left(1 /\left(1-e^{\left.-r_{0}\right)}\right)+B_{1}\left(e^{r_{1}} /\left(1-e^{r_{1}}\right)\right)\right. \\
& \leqq 2 \max \left(B_{0}, B_{1}\right) \max \left\{1 /\left(1-e^{-r_{0}}\right), e^{r_{1}} /\left(1-e^{r_{1}}\right)\right\} \\
& =2 \max \left\{1 /\left(1-e^{-r_{0}}\right), e^{r_{1}} /\left(1-e^{r_{1}}\right)\right\}\|f\|_{W_{x}} .
\end{aligned}
$$


Thus $T_{1}$ is bounded. Similarly, one shows that $T_{2}$ is bounded. The linearity of the maps is easily verified. It is clear that $T_{1}=T_{2}$ if $X_{0}=Y_{0}$ and $X_{1}=Y_{1}$. We see too that

$$
\begin{aligned}
\left(L T_{1}\right) f & =L\left(\sum_{-\infty}^{\infty} f(n)\right)=\sum_{-\infty}^{\infty} L(f(n))=\sum_{-\infty}^{\infty}(L f)(n) \\
& =T_{2}(L f)=T_{2}\left(L^{\prime}(f)\right)=\left(T_{2} L^{\prime}\right) f .
\end{aligned}
$$

Thus, $L T_{1}=T_{2} L^{\prime}$, all three conditions are satisfied, and we have constructed still another standard interpolation space.

III. The generalized duality theorem. Calderón, Lions-Peetre, and Schechter have all used elegant machinery to construct dual spaces for their respective interpolation spaces, $\left(X_{0}, X_{1}\right)_{s}, S\left(p_{0}, r_{0}, X_{0} ; p_{1}, r_{1}, X_{1}\right)$ and $\left(X_{0}, X_{1}\right)_{T}$ which were considered in $\S$ II. All of these dual spaces proved to be interpolation spaces themselves.

In this section we establish a general procedure for locating the dual space of a given generalized interpolation space. This dual space proves also to be a related generalized interpolation space.

Subsequently, we show that the Calderón, Lions-Peetre and Schechter duality theorems are all special cases of our generalized duality theorem.

The following lemma will be useful in the sequel.

Lemma. Suppose $A$ is a generalized interpolation space in $X$. (See definition in §II.) Then $A$ has a continuous embedding in $X$. In particular, if $A$ is a generalized interpolation space in $X$ relative to the mapping $T$ and space $C$, then for each element $a$ in $A,\|a\|_{X} \leqq\|T\|\|a\|_{A}$.

Proof. Let $a$ be a member of $A$. Let $f$ be any member of $C$ with the property $T(f)=a . T$ is a bounded linear operator from $C$ to $X$. Therefore,

$$
\|a\|_{X}=\|T(f)\|_{X} \leqq\|T\|\|f\|_{C} .
$$

Thus,

$$
\|a\|_{X} \leqq \inf _{f \in C}\left\{\|T\|\|f\|_{C} \mid T(f)=a\right\}
$$

Therefore,

$$
\begin{aligned}
\|a\|_{X} & \leqq\|T\| \inf _{f \in C}\left\{\|f\|_{C} \mid T(f)=a\right\} \\
& =\|T\|\|a\|_{A} .
\end{aligned}
$$

Theorem 3.1 (Generalized Duality Theorem). (1) Let $A$ be a generalized interpolation space in $X$ relative to $T_{1}$ and $C$. 
(2) Let $D$ be a normed vector space which is also a dense subspace of $A$, and let $B$ be an interpolation space in $D^{*}$, the dual of $D$ with respect to the $D$-norm, relative to $T_{2}$ and $C^{*}$, the dual of $C$.

(3) Each $f$ in $B$ is also in $D^{*}$ and is therefore a bounded linear functional on $D$ with respect to the D-norm. Suppose in addition that each $f$ in $B$ is also a bounded linear functional on $D$ with respect to the norm of $A$.

Observe now that since $D$ is dense in $A$ each $f$ in $B$ has a unique continuous extension $f^{\prime}$ in $A^{*}$, the dual of $A$.

Another pertinent observation is that for each bounded linear functional $L$ in $A^{*}$, the composition mapping $L T_{1}$ is a member of $C^{*}$. Thus, the image $T_{2}\left(L T_{1}\right)$ of $L T_{1}$ under $T_{2}$ is a well-defined element of $B$.

(4) Suppose that the mappings $T_{1}$ and $T_{2}$ are such that for each $L$ in $A^{*}, T_{2}\left(L T_{1}\right)=f$, where $f$ satisfies $f^{\prime}=L$.

Then $A^{*}$ is isomorphic to $B$.

Proof. Let the mapping $F$ of $A^{*}$ into $B$ be defined by $F(L)=T_{2}\left(L T_{1}\right)$.

(i) $F$ is linear. For let $L_{1}, L_{2}$ be members of $A^{*}$, and $c_{1}, c_{2}$, complex numbers. Then

$$
\begin{aligned}
F\left(c_{1} L_{1}+c_{2} L_{2}\right) & =T_{2}\left(\left(c_{1} L_{1}+c_{2} L_{2}\right) T_{1}\right) \\
& =T_{2}\left(\left(c_{1} L_{1}\right) T_{1}+\left(c_{2} L_{2}\right) T_{1}\right) \\
& =T_{2}\left(c_{1}\left(L_{1} T_{1}\right)+c_{2}\left(L_{2} T_{1}\right)\right) \\
& =c_{1} T_{2}\left(L_{1} T_{1}\right)+c_{2} T_{2}\left(L_{2} T_{1}\right) \\
& =c_{1} F\left(L_{1}\right)+c_{2} F\left(L_{2}\right) .
\end{aligned}
$$

(ii) $F$ is one-to-one. For let $L_{1}, L_{2}$ be members of $A^{*}$. Suppose $F\left(L_{1}\right)=f_{1}$, and $F\left(L_{2}\right)=f_{2}$. Then by assumption (4) $f_{1}^{\prime}=L_{1}$ and $f_{2}^{\prime}=L_{2}$. Thus if $F\left(L_{1}\right)=F\left(L_{2}\right)$, $f_{1}=f_{2}$. Therefore $f_{1}^{\prime}=f_{2}^{\prime}$. Hence $L_{1}=L_{2}$.

(iii) $F$ is onto. For by assumptions (3) and (4) together with previous remarks in the hypothesis, we conclude that for each $f$ in $B, f^{\prime}$ is in $A^{*}$ and $T_{2}\left(f^{\prime} T_{1}\right)=f$.

(iv) $F$ is bounded. For let $L$ be an element in $A^{*}$. Then

$$
\begin{aligned}
\|F(L)\|_{B} & =\left\|T_{2}\left(L T_{1}\right)\right\|_{B} \\
& \leqq\left\|L T_{1}\right\|_{C^{*}} \quad(\text { by definition of the norm in } B) .
\end{aligned}
$$

Now for each $g$ in $C$,

$$
\begin{aligned}
\left|\left(L T_{1}\right) g\right| & =\left|L\left(T_{1}(g)\right)\right| \\
& \leqq\|L\|_{A^{*}}\left\|T_{1}(g)\right\|_{A} \\
& \leqq\|L\|_{A^{*}}\|g\|_{C} \quad(\text { by definition of norm in } A) .
\end{aligned}
$$


Therefore

$$
\left\|L T_{1}\right\|_{C^{*}} \leqq\|L\|_{A^{*}} \text { and }\|F(L)\|_{B} \leqq\|L\|_{A^{*}}
$$

Since $B$ and $A^{*}$ are Banach spaces, and $F$ is a one-to-one, onto, bounded linear operator from $A^{*}$ to $B$, we conclude by the classical Bounded Inverse Theorem that $F$ is an isomorphism from $A^{*}$ to $B$.

REMARK. If for each $f$ in $B,\left\|f^{\prime}\right\|_{A^{*}} \leqq\|f\|_{B}$, then $F$ is an isometric isomorphism. This is clear since, by the last inequality in the proof of (iv),

$$
\|f\|_{B}=\left\|F\left(f^{\prime}\right)\right\|_{B} \leqq\left\|f^{\prime}\right\|_{A^{*}} .
$$

Thus for each $L$ in $A^{*},\|F(L)\|_{B}=\|L\|_{A^{*}}$.

We now define a second Calderón interpolation space which appears in the statement of his duality theorem. Let $H^{\prime}\left(X_{0}, X_{1}\right)$ be the set of functions $f$ mapping the closure of the open strip $S$ between 0 and 1 into $X_{0}+X_{1}$ which satisfy the following:

(1) $f$ is analytic in $S$.

(2) $f$ is continuous in the closure of $S$.

(3) There exists a constant $K$ such that

$$
\|f(z)\|_{x_{0}+X_{1}}=K(1+|z|) .
$$

(4) For all pairs of real numbers $t_{1}, t_{2}, f\left(i t_{1}\right)-f\left(i t_{2}\right)$ is a member of $X_{0}$ and $f\left(1+i t_{1}\right)-f\left(1+i t_{2}\right)$ is a member of $X_{1}$.

(5) $\max \left\{\sup _{t_{2} \neq t_{1}}\left\|\left(f\left(i t_{2}\right)-f\left(i t_{1}\right)\right) /\left(t_{2}-t_{1}\right)\right\|_{x_{0}}, \sup _{t_{2} \neq t_{1}}\left\|\left(f\left(1+i t_{2}\right)-f\left(1+i t_{1}\right)\right) /\left(t_{2}-t_{1}\right)\right\|_{x_{1}}\right\}$

is finite.

$H\left(X_{0}, X_{1}\right)$ reduced modulo the constant functions is a Banach space under the norm

$$
\begin{aligned}
\|f\|_{H\left(X_{0}, X_{1}\right)}=\max \left\{\sup _{t_{2}, t_{1}}\left\|\left(f\left(i t_{2}\right)-f\left(i t_{1}\right)\right) /\left(t_{2}-t_{1}\right)\right\|_{X_{0}},\right. \\
\left.\sup _{t_{2}, t_{1}}\left\|\left(f\left(1+i t_{2}\right)-f\left(1+i t_{1}\right)\right) /\left(t_{2}-t_{1}\right)\right\|_{X_{1}}\right\} \quad\left(t_{2} \neq t_{1}\right) .
\end{aligned}
$$

See $[2$, p. 130].

Fix $s$ between 0 and 1, and let $\left(X_{0}, X_{1}\right)^{s}$ be the set of all $x$ in $X_{0}+X_{1}$ such that $x=f^{\prime}(s)$, where $f$ is a member of $H^{\prime}\left(X_{0}, X_{1}\right) .\left(X_{0}, X_{1}\right)^{s}$ is a Banach space under the norm

$$
\|x\|_{\left(X_{0}, X_{1}\right)^{s}}=\inf _{f \in H^{\prime}\left(X_{0}, X_{1}\right)}\left\{\|f\|_{H^{\prime}\left(X_{0}, X_{1}\right)} \mid f^{\prime}(s)=x\right\} .
$$

See $[2$, p. 130]. 
Let $T$ mapping $H^{\prime}\left(X_{0}, X_{1}\right)$ into $X_{0}+X_{1}$ be defined by $T(f)=f^{\prime}(s)$.

THEOREM 3.2. $\left(X_{0}, X_{1}\right)^{\text {s }}$ is a generalized interpolation space in $X_{0}+X_{1}$ relative to $T$ and $H^{\prime}\left(X_{0}, X_{1}\right)$.

Proof. It is enough to show that the mapping $T$ above is a bounded linear operator. Let $f, g$ be members of $H^{\prime}\left(X_{0}, X_{1}\right)$ and $c, d$ complex numbers. Then

$$
T(c f+d g)=(c f+d g)^{\prime}(s)=\left(c f^{\prime} s\right)+d g^{\prime} s=c T(f)+d T(g) .
$$

Therefore $T$ is a linear operator. Let $f$ be a member of $H^{\prime}\left(X_{0}, X_{1}\right)$. Then

$$
\begin{aligned}
& \|T(f)\|_{X_{0}+X_{1}}=\left\|f^{\prime}(s)\right\|_{X_{0}+X_{1}} \\
& \leqq \max \left\{\sup _{t_{2}, t_{1}}\left\|\left(f\left(i t_{2}\right)-f\left(i t_{1}\right)\right) /\left(t_{2}-t_{1}\right)\right\|_{X_{0}+X_{1}},\right. \\
& \qquad \sup _{t_{2}, t_{1}}\left\|\left(f\left(1+i t_{2}\right)-f\left(1+i t_{1}\right)\right) /\left(t_{2}-t_{1}\right)\right\|_{\left.X_{0}+X_{1}\right\}} \quad\left(t_{2} \neq t_{1}\right) \\
& \quad \text { (by maximum modulus theorem for the strip) } \\
& =\max \left\{\sup _{t_{2} \neq t_{1}}\left\|\left(f\left(i t_{2}\right)-f\left(i t_{1}\right)\right) /\left(t_{2}-t_{1}\right)\right\|_{X_{0}},\right. \\
& \left.\sup _{t_{2} \neq t_{1}}\left\|\left(f\left(1+i t_{2}\right)-f\left(1+i t_{1}\right)\right) /\left(t_{2}-t_{1}\right)\right\|_{X_{1}}\right\} \\
& \left.\quad \text { (by definition of norm in } X_{0}+X_{1}\right)
\end{aligned}
$$

Thus, $T$ is bounded.

Calderón Duality Theorem. Suppose that $X_{0} \cap X_{1}$ is dense in both $X_{0}$ and $X_{1}$. Then $\left(X_{0}, X_{1}\right)_{s}^{*}$ is congruent (isometrically isomorphic) to $\left(X_{0}^{*}, X_{1}^{*}\right)^{s}$ where $s$ is a fixed real number between 0 and 1 .

Proof. We will show that all of the assumptions of the above generalized duality theorem are satisfied, and the conclusion of the Calderón duality theorem will follow as a consequence.

(1) $\left(X_{0}, X_{1}\right)_{s}$ is a generalized interpolation space in $X_{0}+X_{1}$ relative to $T_{1}$ and $H\left(X_{0}, X_{1}\right)$, where $T_{1}$ is defined by $T_{1}(f)=f(s)$. See Theorem 2.2.

(2) $H^{\prime}\left(X_{0}^{*}, X_{1}^{*}\right)$ is congruent to $H\left(X_{0}, X_{1}\right)^{*}\left[2\right.$, pp. 148-156]. $X_{0} \cap X_{1}$ is dense in $\left(X_{0}, X_{1}\right)_{s}$ [2, p. 116]. Under the norm

$$
\|x\|_{X_{0} \cap X_{1}}=\max \left\{\|x\|_{x_{0}},\|x\|_{x_{1}}\right\}
$$

$X_{0} \cap X_{1}$ is a Banach space [2, p. 114]. It is clear that under this norm $X_{0} \cap X_{1}$ has a continuous embedding in each of $X_{0}$ and $X_{1}$. Thus, since $X_{0} \cap X_{1}$ is dense in both $X_{0}$ and $X_{1}$, each of $X_{0}^{*}, X_{1}^{*}$ has a continuous embedding in $\left(X_{0} \cap X_{1}\right)^{*}$. It is a consequence of the definition of the norm in $X_{0}^{*}+X_{1}^{*}$ that $X_{0}^{*}+X_{1}^{*}$ also has a continuous embedding in $\left(X_{0} \cap X_{1}\right)^{*}$. Finally, we conclude that $\left(X_{0}^{*}, X_{1}^{*}\right)^{s}$ is a 
generalized interpolation space in $\left(X_{0} \cap X_{1}\right)^{*}$ relative to $T_{2}$ and $H\left(X_{0}, X_{1}\right)^{*}$ where $T_{2}$ is defined by $T_{2}(f)=f^{\prime}(s)$, since by Theorem $3.2,\left(X_{0}^{*}, X_{1}^{*}\right)^{s}$ is a generalized interpolation space in $X_{0}^{*}+X_{1}^{*}$ relative to $T_{2}$ and $H^{\prime}\left(X_{0}^{*}, X_{1}^{*}\right)$.

(3) For each $f$ in $\left(X_{0}^{*}, X_{1}^{*}\right)^{s}, f$ is a bounded linear functional on $X_{0} \cap X_{1}$ with respect to the norm of $\left(X_{0}, X_{1}\right)_{s}$ satisfying

$$
\|f\|_{\left(X_{0}, X_{1}\right)_{s}^{*}} \leqq\|f\|_{\left(X_{0}^{*}, x_{1}^{*}\right)^{s}} \quad[2, \text { p. } 121]
$$

(4) For each $L$ in $\left(X_{0}, X_{1}\right)_{s}^{*}, L T_{1}$ is a member of $H\left(X_{0}, X_{1}\right)^{*}$ which is congruent to $H^{\prime}\left(X_{0}^{*}, X_{1}^{*}\right)$. Given $g$ in $H\left(X_{0}, X_{1}\right)$,

$$
\left(L T_{1}\right)^{\prime}(s)(g(s))=L(g(s))
$$

where $\left(L T_{1}\right)^{\prime}(s)$ is identified with its extension to $\left(X_{0}, X_{1}\right)_{s}$. See [2, pp. 148-156]. Recalling the definitions of $T_{1}, T_{2}$, one has $\left(T_{2}\left(L T_{1}\right)\right)\left(T_{1}(g)\right)=L\left(T_{1}(g)\right)$ and thus $T_{2}\left(L T_{1}\right)=L$ where $T_{2}\left(L T_{1}\right)$ is identified with its extension to $\left(X_{0}, X_{1}\right)_{s}$.

Thus, the conclusion of the Calderón duality theorem follows as a consequence of the generalized duality theorem and the remark following its proof.

We turn our attention now to the Lions-Peetre duality theorem. Before its statement and proof by use of the generalized duality theorem, we define two new Lions-Peetre interpolation spaces. Subsequently, we exhibit them as significant types of generalized interpolation spaces.

Let $w=w\left(p_{0}, r_{0}, X_{0} ; p_{1}, r_{1}, X_{1}\right)$ with $p_{0}, p_{1}$ real numbers greater than or equal to $1, r_{0}$ positive and $r_{1}$ negative be the set of all pairs of sequences $\left(v_{0 n}, v_{1 n}\right)$ with values in $X_{0}, X_{1}$ respectively, and satisfying

(1) The sequence $e^{n r_{0}} v_{0 n}$ is a member of $l^{p_{0}}\left(X_{0}\right)$ and the sequence $e^{n r_{1} v_{1 n}}$ is a member of $1^{p_{1}}\left(X_{1}\right)$.

(2) There exists $x$ in $X_{0}+X_{1}$ such that, for each integer $n, v_{0 n}+v_{1 n}=x$.

$w$ is a Banach space under the norm

$$
\left\|\left(v_{0 n}, v_{1 n}\right)\right\|_{w}=\max \left\{\left\|e^{n r_{0}} v_{0 n}\right\|_{l^{p_{0}\left(X_{0}\right)}},\left\|e^{n r_{1} v_{1 n}}\right\|_{l^{p_{1}\left(X_{1}\right)}}\right\}
$$

$[6$, p. 17],

$$
s=s\left(p_{0}, r_{0}, X_{0} ; p_{1}, r_{1}, X_{1}\right)=\left\{x \mid x=v_{0 n}+v_{1 n} \text { and }\left(v_{0 n}, v_{1 n}\right) \in w\right\}
$$

is a Banach space under the norm $[6, \mathrm{p} .18]$

$$
\|x\|_{s}=\inf _{x=v_{0 n}+v_{1 n}}\left\{\max \left\{\left\|e^{n r_{0}} v_{0 n}\right\|_{l^{p_{0}\left(X_{0}\right)},}\left\|e^{n r_{1} v_{1 n}}\right\|_{l^{p_{1}}}\left(_{\left.X_{1}\right)}\right\}\right\} .\right.
$$

THEOREM 3.3. $s$ is a generalized interpolation space in $X_{0}+X_{1}$ relative to $T$ and $w$, where $T$ is the mapping from $w$ into $X_{0}+X_{1}$ defined by $T\left(\left(v_{0 n}, v_{1 n}\right)\right)=v_{0 n}+v_{1 n}$.

Proof. One simply has to show that $T$ is a bounded linear operator from $w$ to 
$X_{0}+X_{1}$. Let $\left(v_{0 n}, v_{1 n}\right),\left(w_{0 n}, w_{1 n}\right)$ be members of $w$ and $c, d$ complex numbers. Then

$$
\begin{aligned}
T\left(c\left(v_{0 n}, v_{1 n}\right)+d\left(w_{0 n}, w_{1 n}\right)\right) & =T\left(\left(c v_{0 n}, c v_{1 n}\right)+\left(d w_{0 n}, d w_{1 n}\right)\right) \\
& =T\left(\left(c v_{0 n}+d w_{0 n}, c v_{1 n}+d w_{1 n}\right)\right) \\
& =\left(c v_{0 n}+d w_{0 n}\right)+\left(c v_{1 n}+d w_{1 n}\right) \\
& =c\left(v_{0 n}+v_{1 n}\right)+d\left(w_{0 n}+w_{1 n}\right) \\
& =c T\left(\left(v_{0 n}, v_{1 n}\right)\right)+d T\left(\left(w_{0 n}, w_{1 n}\right)\right) .
\end{aligned}
$$

Thus, $T$ is linear.

Let $\left(v_{0 n}, v_{1 n}\right)$ be a member of $w$. Then

$$
\begin{aligned}
& \left.\left\|T\left(\left(v_{0 n}, v_{1 n}\right)\right)\right\|_{X_{0}+X_{1}}=\left\|v_{0 n}+v_{1 n}\right\|_{X_{0}+X_{1}} \quad \text { (for each } n\right) \\
& =\left\|v_{00}+v_{10}\right\|_{x_{0}+x_{1}} \quad \text { (in particular) } \\
& \leqq\left\|v_{00}\right\|_{x_{0}+x_{1}}+\left\|v_{10}\right\|_{x_{0}+x_{1}} \\
& \leqq\left\|v_{00}\right\|_{x_{0}}+\left\|v_{10}\right\|_{x_{1}} \\
& \leqq\left\|e^{n r_{0} v_{0 n}}\right\|_{l^{p_{0}\left(X_{0}\right)}}+\left\|e^{n r_{1}} v_{1 n}\right\|_{l^{p_{1}\left(X_{1}\right)}} \\
& \leqq 2 \max \left\{\left\|e^{n r_{0} v_{0 n}}\right\|_{l^{p_{0}\left(X_{U}\right)}},\left\|e^{n r_{1} v_{1 n}}\right\|_{l^{p_{1}\left(X_{1}\right)}}\right\} \\
& =2\left\|\left(v_{0 n}, v_{1 n}\right)\right\|_{w} \text {. }
\end{aligned}
$$

Therefore $T$ is bounded.

Let $w^{\prime}=w^{\prime}\left(p_{0}, r_{0}, X_{0} ; p_{1}, r_{1}, X_{1}\right)$ be the set of all sequences $u_{n}$ with values in $X_{0} \cap X_{1}$ such that the sequence $e^{n r_{0}} u_{n}$ is a member of $l^{p_{0}}\left(X_{0}\right)$, and the sequence $e^{n r_{1}} u_{n}$ is a member of $l^{p_{1}}\left(X_{1}\right) . w^{\prime}$ is a Banach space under the norm

$[6$, p. 17] .

$$
\left\|u_{n}\right\|_{w^{\prime}}=\max \left\{\left\|e^{n r_{0}} u_{n}\right\|_{l^{p_{0}\left(X_{0}\right)}},\left\|e^{n r_{1}} u_{n}\right\|_{l^{p_{1}}\left(X_{1}\right)}\right\}
$$

For each sequence $u_{n}$ in $w^{\prime}, \sum_{-\infty}^{\infty} u_{n}$ converges in $X_{0}+X_{1}[6$, p. 17].

Let $s^{\prime}=\left\{x \mid x=\sum_{-\infty}^{\infty} u_{n}\right.$ and $u_{n}$ is a member of $\left.w^{\prime}\right\}$. $s^{\prime}$ is a Banach space under the norm

$$
\|x\|_{s^{\prime}}=\inf _{x=\sum_{-\infty}^{\infty} u_{n}}\left\{\max \left\|e^{n r_{0}} u_{n}\right\|_{l^{p_{0}\left(X_{0}\right)}},\left\|e^{n r_{1}} u_{n}\right\|_{l^{p_{1}}\left(X_{1}\right)}\right\}
$$

$[6$, p. 17]

Let $T$ mapping $w^{\prime}$ into $X_{0}+X_{1}$ be defined by $T\left(u_{n}\right)=\sum_{-\infty}^{\infty} u_{n}$.

THEOREM 3.4. $s^{\prime}$ is a generalized interpolation space in $X_{0}+X_{1}$ relative to $T$ and $w^{\prime}$.

Proof. We proceed to show that $T$ is a bounded linear mapping from $w^{\prime}$ into $X_{0}+X_{1}$. Let $u_{n}, w_{n}$ be members of $w^{\prime}$, and $c, d$ complex numbers. Then

$$
\begin{aligned}
T\left(c u_{n}+d w_{n}\right) & =\sum_{-\infty}^{\infty}\left(c u_{n}+d w_{n}\right)=\sum_{-\infty}^{\infty} c u_{n}+\sum_{-\infty}^{\infty} d w_{n} \\
& =c \sum_{-\infty}^{\infty} u_{n}+d \sum_{-\infty}^{\infty} w_{n}=c T\left(u_{n}\right)+d T\left(w_{n}\right) .
\end{aligned}
$$

Therefore $T$ is linear. 
Let $u_{n}$ be a member of $w^{\prime}$. Then

$$
\begin{aligned}
& \left\|T\left(u_{n}\right)\right\|_{X_{0}+X_{1}}=\left\|\sum_{-\infty}^{\infty} u_{n}\right\|_{X_{0}+X_{1}} \\
& \leqq \sum_{-\infty}^{\infty}\left\|u_{n}\right\|_{x_{0}+x_{1}} \\
& \leqq \sum_{0}^{\infty}\left\|u_{n}\right\|_{x_{0}+x_{1}}+\sum_{-\infty}^{0}\left\|u_{n}\right\|_{x_{0}+x_{1}} \\
& \leqq \sum_{0}^{\infty}\left\|u_{n}\right\|_{x_{0}}+\sum_{-\infty}^{0}\left\|u_{n}\right\|_{x_{1}} \\
& =\sum_{0}^{\infty}\left\|e^{-n r_{0}}\left(e^{n r_{0}} u_{n}\right)\right\|_{X_{0}}+\sum_{-\infty}^{0}\left\|e^{-n r_{1}}\left(e^{n r_{1}} u_{n}\right)\right\|_{X_{1}} \\
& \leqq\left\|e^{-n r_{0}}\right\|_{l} q_{0(0, \infty)}\left\|e^{n r_{0}} u_{n}\right\|_{l} p_{0\left(X_{0}\right)} \\
& +\left\|e^{-n r_{1}}\right\|_{l^{\left.q_{1}-\infty, 0\right)}}\left\|e^{n r_{1}} u_{n}\right\|_{l^{p_{1}\left(X_{1}\right)}} \quad\left(1 / q_{i}+1 / p_{i}=1 ; i=0,1\right) \\
& \leqq 2 \max \left\{\left\|e^{-n r_{0}}\right\|_{l^{q_{0}(0, \infty)}},\left\|e^{-n r_{1}}\right\|_{l^{q_{1}}(-\infty, 0)}\right\} \\
& \cdot \max \left\{\left\|e^{n r_{0}} u_{n}\right\|_{l} l_{0\left(X_{0}\right)},\left\|e^{n r_{1}} u_{n}\right\|_{l^{p_{1}}\left(X_{1}\right)}\right\} \\
& =2 \max \left\{\left\|e^{-n r_{0}}\right\|_{l^{q_{0}(0, \infty)}},\left\|e^{-n r_{1}}\right\|_{l^{q_{1}}(-\infty, 0)}\right\}\left\|u_{n}\right\|_{w^{\prime}} \text {. }
\end{aligned}
$$

Therefore $T$ is bounded.

Lions-Peetre Duality Theorem. (1) Suppose $X_{0} \cap X_{1}$ is dense in both $X_{0}$ and $X_{1}$.

(2) For $i=0,1$, let $p_{i}$ satisfy $1 \leqq p_{i}<\infty$, and let $q_{i}$ satisfy $1 / p_{i}+1 / q_{i}=1$.

(3) Suppose the real numbers $r_{0}, r_{1}$ satisfy $r_{0} r_{1}$ is less than 0 (without loss of generality we assume that $r_{0}$ is greater than 0 and $r_{1}$ is less than 0 ).

Then $s\left(p_{0}, r_{0}, X_{0} ; p_{1}, r_{1}, X_{1}\right)^{*}$ is isomorphic to $s^{\prime}\left(q_{0},-r_{0}, X_{0}^{*} ; q_{1},-r_{1}, X_{1}^{*}\right)$.

Proof. We will show that the four hypotheses of the generalized duality theorem follow from the assumptions of the Lions-Peetre duality theorem, and the conclusion of the Lions-Peetre duality theorem will thereby follow.

(1) $s\left(p_{0}, r_{0}, X_{0} ; p_{1}, r_{1}, X_{1}\right)$ is a generalized interpolation space in $X_{0}+X_{1}$ relative to $T_{1}$ and $w$ where $T_{1}$ is defined by

$$
T_{1}\left(\left(v_{0 n}, v_{1 n}\right)\right)=v_{0 n}+v_{1 n}
$$

for each pair of sequences $\left(v_{0 n}, v_{1 n}\right)$ in $w=w\left(p_{0}, r_{0}, X_{0} ; p_{1}, r_{1}, X_{1}\right)$. See Theorem 3.3.

(2) (i) $w\left(p_{0}, r_{0}, X_{0} ; p_{1}, r_{1}, X_{1}\right)^{*}$ is congruent to $w^{\prime}\left(q_{0},-r_{0}, X_{0}^{*} ; q_{1},-r_{1}, X_{1}^{*}\right)$ [6, pp. 21-25].

(ii) $X_{0} \cap X_{1}$ is dense in $s\left(p_{0}, r_{0}, X_{0} ; p_{1}, r_{1}, X_{1}\right)$. See [6, p. 22].

(iii) $s^{\prime}\left(q_{0},-r_{0}, X_{0}^{*} ; q_{1},-r_{1}, X_{1}^{*}\right)$ is a generalized space of interpolation in $X_{0}^{*}+X_{1}^{*}$ relative to $w^{\prime}\left(q_{0},-r_{0}, X_{0}^{*} ; q_{1},-r_{1}, X_{1}^{*}\right)$, and $T_{2}$ where $T_{2}$ is defined by $T_{2}\left(u_{n}\right)=\sum_{-\infty}^{\infty} u_{n}$. See Theorem 3.4. 
(iv) $X_{0} \cap X_{1}$ with the standard norm defined in the proof of the Calderon theorem is as before a Banach space with continuous embeddings in $X_{0}, X_{1}$, respectively. Thus, since $X_{0} \cap X_{1}$ is dense in both $X_{0}$ and $X_{1}$, each of $X_{1}^{*}, X_{0}^{*}$ and $X_{0}^{*}+X_{1}^{*}$ has a continuous embedding in $\left(X_{0} \cap X_{1}\right)^{*}$.

(i), (ii), (iii) and (iv) clearly imply that $s^{\prime}\left(q_{0},-r_{0}, X_{0}^{*} ; q_{1},-r_{1}, X_{1}^{*}\right)$ is a generalized interpolation space in $\left(X_{0} \cap X_{1}\right)^{*}$ relative to $T_{2}$ and $w\left(p_{0}, r_{0}, X_{0} ; p_{1}, r_{1}, X_{1}\right)^{*}$.

(3) Given $f$ in $s^{\prime}\left(q_{0},-r_{0}, X_{0}^{*} ; q_{1},-r_{1}, X_{1}^{*}\right)$, then there exists $u_{n}$ in

$$
w^{\prime}\left(q_{0},-r_{0}, X_{0}^{*} ; q_{1},-r_{1}, X_{1}^{*}\right)
$$

such that

$$
f=\sum_{-\infty}^{\infty} u_{n}
$$

With this identification, $f$ is shown by direct computation to be a bounded linear functional on $X_{0} \cap X_{1}$ relative to the norm of $s\left(p_{0}, r_{0}, X_{0} ; p_{1}, r_{1}, X_{1}\right)$. See [6, pp. 24-25].

(4) For each $L$ in $s\left(p_{0}, r_{0}, X_{0} ; p_{1}, r_{1}, X_{1}\right)^{*}, L T_{1}$ is a member of

$$
w\left(p_{0}, r_{0}, X_{0} ; p_{1}, r_{1}, X_{1}\right)^{*} \text {. }
$$

Recall that $w\left(p_{0}, r_{0}, X_{0} ; p_{1}, r_{1}, X_{1}\right)^{*}$ is congruent to $w^{\prime}\left(q_{0},-r_{0}, X_{0}^{*} ; q_{1},-r_{1}, X_{1}^{*}\right)$. Thus, $L T_{1}$ may be identified with a member $\left(L T_{1}\right)_{n}$ of $w^{\prime}\left(q_{0},-r_{0}, X_{0}^{*} ; q_{1},-r_{1}, X_{1}^{*}\right)$. For each member $\left(v_{0 n}, v_{i n}\right)$ of $w\left(p_{0}, r_{0}, X_{0} ; p_{1}, r_{1}, X_{1}\right)$,

$$
\begin{aligned}
L\left(v_{0 n}+v_{i n}\right) & =\sum_{-\infty}^{\infty}\left\{\left(L T_{1}\right)_{n}\left(v_{0 n}+v_{i n}\right)\right\} \quad[6, \text { pp. 21-25] } \\
& =\left(\sum_{-\infty}^{\infty}\left(L T_{1}\right)_{n}\right)\left(v_{0 n}+v_{1 n}\right)
\end{aligned}
$$

where $\sum_{-\infty}^{\infty}\left(L T_{1}\right)_{n}$ is identified with its extension to $s\left(p_{0}, r_{0}, X_{0} ; p_{1}, r_{1}, X_{1}\right)$.

Hence, $L=\sum_{-\infty}^{\infty}\left(L T_{1}\right)_{n}$. Recalling the definition of the operator $T_{2}$, it is clear that

$$
L=T_{2}\left(\left(L T_{1}\right)_{n}\right)=T_{2}\left(L T_{1}\right),
$$

where $T_{2}\left(L T_{1}\right)$ is identified with its extension to $s\left(p_{0}, r_{0}, X_{0} ; p_{1}, r_{1}, X_{1}\right)$. The conclusion of the Lions-Peetre duality theorem now follows from the generalized duality theorem.

REMARK. The spaces $s\left(p_{0}, r_{0}, X_{0} ; p_{1}, r_{1}, X_{1}\right), s^{\prime}\left(p_{0}, r_{0}, X_{0} ; p_{1}, r_{1}, X_{1}\right)$ and $S\left(p_{0}, r_{0}, X_{0} ; p_{1}, r_{1}, X_{1}\right)$ are mutually congruent, as indeed are the spaces $w\left(p_{0}, r_{0}, X_{0} ; p_{1}, r_{1}, X_{1}\right), w^{\prime}\left(p_{0}, r_{0}, X_{0} ; p_{1}, r_{1}, X_{1}\right)$ and $W\left(p_{0}, r_{0}, X_{0} ; p_{1}, r_{1}, X_{1}\right)$. Hence, given the hypotheses of the Lions-Peetre duality here considered, one has the result that $S\left(p_{0}, r_{0}, X_{0} ; p_{1}, r_{1}, X_{1}\right)^{*}$ is isomorphic to the Banach space $S\left(q_{0},-r_{0}, X_{0}^{*} ; q_{1},-r_{1}, X_{1}^{*}\right)$. See [6]. 
Before stating the Schechter duality theorem, and establishing that it too is a special case of the generalized duality theorem, we introduce a new Schechter interpolation space.

Let $\left(X_{0}, X_{1}\right)^{\prime T}$ be the set of all $x$ in $X_{0}+X_{1}$ such that

(i) $x T=f T$,

where $T$ is a nontrivial distribution with compact support and $f$ is a member of $H^{\prime}\left(X_{0}, X_{1}\right)$.

$\left(X_{0}, X_{1}\right)^{\prime T}$ is a Banach space under the norm

$$
\|x\|_{\left(X_{0}, X_{1}\right)^{\prime T}}=\inf _{f^{\prime} T=x T}\|f\|_{H^{\prime}\left(X_{0}, X_{1}\right)} \quad[11, \text { p. 120]. }
$$

Let $S^{\prime}\left(X_{0}, X_{1}\right)$ be the set of all $f$ in $H^{\prime}\left(X_{0}, X_{1}\right)$ for which there exists $x$ in $X_{0}+X_{1}$ satisfying $f^{\prime} T=x T . S^{\prime}\left(X_{0}, X_{1}\right)$ is clearly a subspace of $H^{\prime}\left(X_{0}, X_{1}\right)$. We will show that it is closed in $H^{\prime}\left(X_{0}, X_{1}\right)$, and thus is a Banach space. Let $f_{n}$ be a sequence in $S^{\prime}\left(X_{0}, X_{1}\right)$ which converges to $f$ in $H^{\prime}\left(X_{0}, X_{1}\right)$. Thas compact support, therefore there exists constant $K$ such that, for each $\phi$ in $H(C, C)$,

$$
\left\|T\left(f_{n}^{\prime} \phi-f^{\prime} \phi\right)\right\|_{X_{0}+X_{1}} \leqq K\|\phi\|_{H(C, C)}\left\|f_{n}-f\right\|_{H^{\prime}\left(X_{0}, X_{1}\right)} .
$$

Thus, for each $\phi$ in $H(C, C), T\left(f_{n}^{\prime} \phi\right)$ converges to $T\left(f^{\prime} \phi\right)$ in $X_{0}+X_{1}$. On the other hand, the convergence of $f_{n}$ to $f$ in $H^{\prime}\left(X_{0}, X_{1}\right)$ implies that $\left\|f_{n}-f_{m}\right\|_{H^{\prime}\left(X_{0}, X_{1}\right)}$ converges to 0 as $m, n$ go to infinity. For each $f_{n}$ in $S^{\prime}\left(X_{0}, X_{1}\right)$ there exists $x_{n}$ in $X_{0}+X_{1}$ such that $f_{n}^{\prime} T=x_{n} T$. Thus,

$$
\begin{aligned}
\left\|x_{n}-x_{m}\right\|_{X_{0}+x_{1}}|T \phi| & =\left\|T\left(x_{n} \phi-x_{m} \phi\right)\right\|_{X_{0}+x_{1}} \\
& =\left\|T\left(f_{n}^{\prime} \phi-f_{m}^{\prime} \phi\right)\right\|_{X_{0}+x_{1}} \\
& \leqq K_{1}\|\phi\|_{H(C, C)}\left\|f_{n}-f_{m}\right\|_{H^{\prime}\left(X_{0}, x_{1}\right)} .
\end{aligned}
$$

Hence, $\left\|x_{n}-x_{m}\right\|_{X_{0}+X_{1}}|T \phi|$ converges to 0 as $m, n$ go to infinity for each $\phi$ in $H(C, C) . T$ is nontrivial, that is there exists $\phi$ in $H(C, C)$ such that $T \phi \neq 0$. Therefore $\left\|x_{n}-x_{m}\right\|_{X_{0}+x_{1}}$ converges to 0 as $m, n$ go to infinity. $X_{0}+X_{1}$ is complete. Thus there is an $x$ in $X_{0}+X_{1}$ such that the sequence $x_{n}$ converges to $x$ in $X_{0}+X_{1}$. Therefore for each $\phi$ in $H(C, C), x_{n} T \phi$ converges to $x T \phi$. Since $x_{n} T \phi=T\left(f_{n}^{\prime} \phi\right)$, and as shown above $T\left(f_{n}^{\prime} \phi\right)$ converges to $T\left(f^{\prime} \phi\right), x T \phi=T\left(f^{\prime} \phi\right)$. Thus $x T=f^{\prime} T$. Hence $f$ is a member of $S^{\prime}\left(X_{0}, X_{1}\right)$, and it is clear that $S^{\prime}\left(X_{0}, X_{1}\right)$ is closed.

Let the mapping $T_{1}$ of $S^{\prime}\left(X_{0}, X_{1}\right)$ into $X_{0}+X_{1}$ be defined by

$$
T_{1}(f)=x, \quad \text { where } f^{\prime} T=x T .
$$

This mapping is well defined for suppose $x_{1} T=x_{2} T$. Then for each $\phi$ in $H(C, C)$, $x_{1}(T \phi)=x_{2}(T \phi)$. Therefore $x_{1}=x_{2}$ since $T$ is nontrivial.

THEOREM 3.5. $\left(X_{0}, X_{1}\right)^{\prime T}$ is a generalized interpolation space in $X_{0}+X_{1}$ relative to $T_{1}$ and $S^{\prime}\left(X_{0}, X_{1}\right)$.

Proof. It is sufficient to show that $T_{1}$ is a bounded linear operator from $S^{\prime}\left(X_{0}, X_{1}\right)$ to $X_{0}+X_{1}$. Let $f, g$ be members of $S^{\prime}\left(X_{0}, X_{1}\right)$ and $c, d$ complex numbers. Suppose 
$T_{1}(f)=x_{1}$, and $T_{1}(g)=x_{2}$. Then $T_{1}(c f+d g)=x$ where $x$ satisfies $\left(c f^{\prime}+d g^{\prime}\right) T=x T$. Thus,

$$
\begin{aligned}
\left(c f^{\prime}\right) T+\left(d g^{\prime}\right) T & =x T, & c\left(f^{\prime} T\right)+d\left(g^{\prime} T\right) & =x T, \\
c\left(x_{1} T\right)+d\left(x_{2} T\right) & =x T, & \left(c x_{1}+d x_{2}\right) T & =x T .
\end{aligned}
$$

Therefore, $c x_{1}+d x_{2}=x$. Hence,

$$
c\left(T_{1}(f)\right)+d\left(T_{1}(g)\right)=T_{1}(c f+d g) .
$$

The linearity of $T_{1}$ is now established.

$T_{1}$ is continuous, for suppose $f_{n}$ converges to $f$ in $S^{\prime}\left(X_{0}, X_{1}\right)$. Let $x_{n}=T_{1}\left(f_{n}\right)$, and $x=T_{1}(f) . T$ has compact support. Therefore, there exists a constant $k$ such that, for each $\phi$ in $H(C, C)$,

$$
\left\|T\left(f_{n}^{\prime} \phi\right)-T\left(f^{\prime} \phi\right)\right\|_{X_{0}+X_{1}} \leqq K\|\phi\|_{H(C, C)}\left\|f_{n}-f\right\|_{S^{\prime}\left(X_{0}, x_{1}\right)} .
$$

Therefore $\left\|T\left(f_{n}^{\prime} \phi\right)-T\left(f^{\prime} \phi\right)\right\|_{x_{0}+x_{1}}$ converges to 0 as $n$ goes to infinity. However,

$$
\begin{aligned}
\left\|T\left(f_{n}^{\prime} \phi\right)-T\left(f^{\prime} \phi\right)\right\|_{X_{0}+x_{1}} & =\left\|x_{n}(T \phi)-x(T \phi)\right\|_{x_{0}+x_{1}} \\
& =\left\|x_{n}-x\right\|_{X_{0}+x_{1}}|T(\phi)| .
\end{aligned}
$$

$T$ is nontrivial on $H(C, C)$, therefore $\left\|x_{n}-x\right\|_{x_{0}+X_{1}}$ converges to 0 in $X_{0}+X_{1}$. Thus, $x_{n}$ converges to $x$ in $X_{0}+X_{1}$. Hence, $T_{1}\left(f_{n}\right)$ converges to $T_{1}(f)$ in $X_{0}+X_{1}$. The continuity of $T_{1}$ is immediate.

Schechter Duality Theorem. (1) Suppose $X_{0} \cap X_{1}$ is dense in both $X_{0}$ and $X_{1}$.

(2) Let $T$ be a two-dimensional distribution with finite support in the open strip.

Then $\left(X_{0}, X_{1}\right)_{T}^{*}$ is isomorphic to $\left(X_{0}^{*}, X_{1}^{*}\right)^{\prime T}$.

Proof. We will show that the Schechter duality theorem is a special case of the generalized duality theorem.

(1) $\left(X_{0}, X_{1}\right)_{T}$ is a generalized interpolation space in $X_{0}+X_{1}$ relative to $T_{1}$ and $H\left(X_{0}, X_{1}\right)$, where $T_{1}$ is defined by $T_{1}(f)=T(f)$. See Theorem 2.4.

(2) (i) $H\left(X_{0}, X_{1}\right)^{*}$ is congruent to $S^{\prime}\left(X_{0}^{*}, X_{1}^{*}\right)[11$, pp. 133-144].

(ii) $X_{0} \cap X_{1}$ is dense in $\left(X_{0}, X_{1}\right)_{T}[11$, p. 145].

(iii) $\left(X_{0}^{*}, X_{1}^{*}\right)^{\prime T}$ is a generalized interpolation space in $X_{0}^{*}+X_{1}^{*}$ relative to $T_{2}$ and $S^{\prime}\left(X_{0}^{*}, X_{1}^{*}\right)$, where $T_{2}$ is defined by $T_{2}(f)=x$, where $f^{\prime} T=x T$. See Theorem 3.5.

(iv) Recall from the proof of the Lions-Peetre duality theorem that each of $X_{1}^{*}, X_{0}^{*}$ and $X_{0}^{*}+X_{1}^{*}$ has a continuous embedding in $\left(X_{0} \cap X_{1}\right)^{*}$. Thus, in view of statements (i), (ii) and (iii), $\left(X_{0}^{*}, X_{1}^{*}\right)^{\prime T}$ is a generalized interpolation space in $\left(X_{0} \cap X_{1}\right)^{*}$ relative to $T_{2}$ and $H\left(X_{0}, X_{1}\right)^{*}$.

(3) For each $L$ in $\left(X_{0}, X_{1}\right)_{T}^{*}, L T_{1}$ is a member of $H\left(X_{0}, X_{1}\right)^{*}$ and hence by (i) above may be identified as an element (also to be denoted by $L T_{1}$ ) in $S^{\prime}\left(X_{0}^{*}, X_{1}^{*}\right)$. Given $g$ in $H\left(X_{0}, X_{1}\right)$,

$$
L(T(g))=T\left(L T_{1}\right)^{\prime}(z)(g(z))=x^{*}(T(g))
$$


where $x^{*}$ is an element of $X_{0}^{*}+X_{1}^{*}$, satisfies $x^{*} T=\left(L T_{1}\right)^{\prime} T$, is identified with its extension to $\left(X_{0}, X_{1}\right)_{T}$, and $T$ is assumed without loss of generality to be a distribution whose support consists of a single point. See [11, pp. 133-144].

By definition $x^{*}=T_{2}\left(L T_{1}\right)$. Hence, $L(T(g))=\left(T_{2}\left(L T_{1}\right)\right)(T(g))$ and $L=T_{2}\left(L T_{1}\right)$, where $T_{2}\left(L T_{1}\right)$ is identified with its extension to $\left(X_{0}, X_{1}\right)_{T}$.

(4) Reversing the procedure which provided a representation for elements of $\left(X_{0}, X_{1}\right)_{T}^{*}$ as members of $\left(X_{0}^{*}, X_{1}^{*}\right)^{\prime T}$, one is able to show that each element of $\left(X_{0}^{*}, X_{1}^{*}\right)^{\prime T}$ gives rise to a bounded linear functional on $\left(X_{0}, X_{1}\right)_{T}$. See [11, p. 143].

The conclusion of the Schechter duality theorem now follows as a consequence of the generalized duality theorem.

\section{BIBLIOGRAPHY}

1. A. P. Calderón, Intermediate spaces and interpolation, Studia Math. (Special Series) No. 1 (1963), 31-34. MR 26 \#5409.

2. - Intermediate spaces and interpolation, the complex method, Studia Math. 24 (1964), 113-190. MR 29 \#5097.

3. N. Dunford and J. T. Schwartz, Linear operators. I. General theory, Pure and Appl. Math., vol. 7, Interscience, New York, 1958. MR 22 \#8302.

4. P. D. Lax, Symmetrizable linear transformations, Comm. Pure Appl. Math. 7 (1954), 633-647. MR 16, 832.

5. J. L. Lions, Une construction d'espaces d'interpolation, C. R. Acad. Sci. Paris 251 (1960), 1853-1855. MR 22 \#9859.

6. J. L. Lions and J. Peetre, Sur une classe d'espaces d'interpolation, Inst. Hautes Études Sci. Publ. Math. No. 19 (1964), 5-68. MR 29 \#2627.

7. E. Magenes, Spazi di interpolazione ed equazioni a derivate parziali, Atti del Settimo Congresso Dell'Unione Matematica Italiana (Genova, 1963), Edizioni Cremonese, Rome, 1965, pp. 134-197. MR 35 \#5925.

8. J. Marcinkiewicz, Sur l'interpolation d'opérateurs, C. R. Acad. Sci. Paris 208 (1939), 12721273.

9. F. Riesz and B. Sz.-Nagy, Functional analysis, 2nd ed., Akad. Kiadó, Budapest, 1953; English transl., Ungar, New York, 1955. MR 15, 132; MR 17, 175.

10. M. Riesz, Sur les maxima des formes bilinéaires et sur les fonctionnelles linéaires, Acta Math. 49 (1926), 465-497.

11. M. Schechter, Complex interpolation, Compositio Math. 18(1967), 117-147. MR 36 \#6927.

12. - General boundary value problems for elliptic partial differential equations, Comm. Pure Appl. Math. 12 (1959), 457-486. MR 23 \#A2626.

13. - On $L^{p}$ estimates and regularity. I, Amer. J. Math. 85 (1963), 1-13. MR 32 \#6051.

14. - On $L^{p}$ estimates and regularity. II, Math. Scand. 13 (1963), 47-69. MR 32 \#6052.

15. A. E. Taylor, Introduction to functional analysis, Wiley, New York, 1958. MR 20 \#5411.

16. E. C. Titchmarsh, Theory of functions, Oxford Univ. Press, London, 1939.

17. A. H. Zemanian, Distribution theory and transform analysis. An introduction to generalized functions, with applications, McGraw-Hill, New York, 1965. MR 31 \#1556.

RUTGERS UNIVERSITY,

New Brunswick, NeW Jersey 08903 